\title{
Numerical Simulations of Fractionated Electrograms and Pathological Cardiac Action Potential*
}

\author{
SIMONA SANFELICI \\ Dipartimento di Studi Economici e Quantitativi, Università di Parma, Via J.F. Kennedy, 6-43100 Parma, Italy
}

(Received 21 March 2001; In final form 11 June 2002)

\begin{abstract}
The aim of this work is twofold. First we focus on the complex phenomenon of electrogram fractionation, due to the presence of discontinuities in the conduction properties of the cardiac tissue in a bidomain model. Numerical simulations of paced activation may help to understand the role of the membrane ionic currents and of the changes in cellular coupling in the formation of conduction blocks and fractionation of the electrogram waveform. In particular, we show that fractionation is independent of $I_{\mathrm{Na}}$ alterations and that it can be described by the bidomain model of cardiac tissue. Moreover, some deflections in fractionated electrograms may give nonlocal information about the shape of damaged areas, also revealing the presence of inhomogeneities in the intracellular conductivity of the medium at a distance.

The second point of interest is the analysis of the effects of space-time discretization on numerical results, especially during slow conduction in damaged cardiac tissue. Indeed, large discretization steps can induce numerical artifacts such as slowing down of conduction velocity, alteration in extracellular and transmembrane potential waveforms or conduction blocks, which are not predicted by the continuous bidomain model. Several possible numerical and physiological explanations of these effects are given. Essentially, the discrete system obtained at the end of the approximation process may be interpreted as a discrete model of the cardiac tissue made up of isopotential cells where the effective intracellular conductivity tensor depends on the space discretization steps; the increase of these steps results in an increase of the effective intracellular resistance and can induce conduction blocks if a certain critical value is exceeded.
\end{abstract}

Keywords: Electrogram fractionation; Bidomain model; Conduction blocks; Cardiac action potential

\section{INTRODUCTION}

A region of "damaged" cardiac tissue is characterized by the presence of cellular membrane subregions having altered excitability and spoiled intracellular conductivity; in these regions the excitation wavefronts propagate in anomalous way and may give rise to conduction blocks, reentries and arrhythmias; in particular, non uniform membrane properties and intercellular coupling may result in local differences in the shape of the action potential and may generate fractionated electrograms with multiple deflections. Such waveforms are frequently recorded in patients affected by coronary diseases, ventricular tachycardia or with chronic infarct. Conduction of excitation in pathological hearts is still not completely understood and represents an interesting open subject of research. Formulation of a mathematical model of activation in pathological cardiac tissue is one tool for gaining insight into the mechanism of conduction abnormalities.

The activation sequence in cardiac tissue is determined by both the active membrane current dynamics and the loading effects of the underlying tissue, due to cellular coupling through gap junctions. Although the accumulation of extracellular potassium and the resulting resting membrane potential depolarization are among the most important determinants of impulse propagation failure in ischemic heart [14,26,34], it seems clear that changes in passive electric properties of cell membrane (i.e. cell-tocell coupling) and discontinuities in effective intracellular resistivity contribute to generate conduction blocks and other rhythm disturbances [27].

In this work, we employ the bidomain model [3] in order to study cardiac excitation in the presence of damaged regions, i.e. regions where the cellular membrane has compromised excitability or altered

*Preliminary results of this study were presented at the XXV International Congress on Electrocardiology, where it received the 2nd prize of the Young Investigators' Award. 
intracellular conductivity. The aim of this work is to simulate electrical phenomena occurring in cardiac diseases, without referring to any specific pathology. We rather want to focus on the possible drawbacks connected with numerical approximations of continuous macroscopic models of cardiac activity, drawbacks that can occur especially during the simulation of pathological conduction, for instance in ischemic or infarcted tissue.

Therefore, we model pathological conditions which may occur in real cardiac tissue, without explicit reference to any specific disease. Our aim is to describe some physiological and numerical aspects connected with the simulation of such abnormal situations related to the excitation process, focusing on the following two topics: the first aspect is the occurrence of electrogram fractionation, i.e. waveforms with multiple deflections, in the presence of non homogeneous distribution of membrane properties; the second one is the effect of numerical discretization during the simulation of slow conduction.

There still exist some controversies in characterizing activation maps related to very slow conduction or to regions with non homogeneous conductivity. In fact, in such situations it is not clear how to identify a set of activation times, from both an empirical and computational point of view [2]. The instant of largest negative derivative $\partial u_{\mathrm{e}} / \partial t$ represents the most common criterion at an experimental level for identifying activation times from electrograms; however, the determination of activation time in the region overlying an infarct is complicated because surviving cells in this region do not have a uniform conduction velocity and the electrograms often appear as fractionated, i.e. with multiple deflections which complicate the choice of the deflection corresponding to local membrane depolarization [35,23]. Fractionated polyphasic electrograms are frequently recorded during mapping studies in patients with coronary artery disease, ventricular tachycardia or chronic, healed myocardial infarction.

The mechanism that produces fractionation is not completely understood. Results from experimental studies suggest that in chronically infarcted ventricular myocardium, slow conduction and fractionated electrograms result from increased coupling resistance between otherwise normal surviving regions of myocardium and not from an abnormality of the membrane action potential [11]. This finding is also confirmed by our numerical simulations. In Ref. [23], it has been shown through computer modeling and also in in vitro experiments that fractionated electrograms can be produced as uniform wavefronts encounter zones of slow conduction produced by increased cell-to-cell coupling resistance. In these conditions, the local activation time may not correspond to the largest or fastest deflection in a polyphasic fractionated electrogram, and this makes the experimental results hard to interpret. In particular, neither criteria on the amplitude nor on the time derivative of the extracellular potential are adequate to identify the activation time and the unique valid criterion is the analysis of the amplitude of the transmembrane potential or sodium current time derivative. More recently, Ellis et al. [10] analyzed the relation between heterogeneous intercellular coupling and electrogram fractionation, using a computer monodomain model of elements grouped into cells heterogeneously coupled through randomly varying gap junctional resistances.

In this work, we analyze the effects of discontinuities of various nature in the passive and active properties of the cardiac tissue on the extracellular electrograms, using a bidomain model of myocardial tissue. This is a very interesting problem, since recurrent discontinuities in cellular coupling at different levels are present also in normal cardiac tissue, and similar phenomena are likely to occur also in normal hearts. In particular, these aspects are analyzed in relation to variations of the discretization parameters and to the effects of such discretization on numerical results.

The plan of the work is the following. In the second section, we define a bidomain model of activation in a simplified 3D wall of anisotropic myocardium. In the third section, we shortly describe the numerical approximation method used and the setting of our simulations. In the fourth section, we present our main results. The first set of simulations, in Section 4.1, is devoted to the analysis of electrogram fractionation in pathological conditions. In Section 4.2, we analyze the effects of discretization in the presence of areas of slow conduction. Indeed, large discretization steps can induce numerical artifacts such as slowing down of conduction velocity, alteration in potential waveforms or conduction blocks, which are not predicted by the continuous bidomain model. These topics are discussed in the fifth section. Finally, some conclusions are provided in the sixth section.

\section{THE MATHEMATICAL MODEL}

A macroscopic model of cardiac activation in a simplified insulated three-dimensional wall of anisotropic myocardium is considered $[3-5,17]$. The model is based on the bidomain representation of the cardiac tissue, in the presence of general anisotropy conditions, fiber rotation laws and Hodgkin-Huxley-type gating equations for the ionic current [13]. It is described by a reaction-diffusion (R-D) system consisting of a semilinear parabolicelliptic system of partial differential equations coupled with an ordinary differential system

$$
\begin{array}{cc}
\chi C_{\mathrm{m}} v_{\mathrm{t}}+\chi I_{\mathrm{ion}}(v, \hat{q})-\operatorname{div} \mathbf{M}_{\mathrm{i}}(\mathbf{x}) \nabla v & \\
=\operatorname{div} \mathbf{M}_{\mathrm{i}}(\mathbf{x}) \nabla u_{\mathrm{e}}+I_{\mathrm{app}}(\mathbf{x}, t) & \text { in } \Omega \times] 0, T[ \\
\operatorname{div} \mathbf{M}(\mathbf{x}) \nabla u_{\mathrm{e}}=-\operatorname{div} \mathbf{M}_{\mathrm{i}}(\mathbf{x}) \nabla v & \text { in } \Omega \times] 0, T[ \\
\hat{q}_{t}+\hat{f}(v, \hat{q})=0 & \text { in } \Omega \times] 0, T[ \\
\mathbf{n}^{T} \mathbf{M}_{\mathrm{i}}(\mathbf{x}) \nabla v=0 \quad \mathbf{n}^{T} \mathbf{M}(\mathbf{x}) \nabla u_{\mathrm{e}}=0 & \text { on } \partial \Omega \times] 0, T[ \\
v(\mathbf{x}, 0)=v_{0} \quad \hat{q}(\mathbf{x}, 0)=\hat{q}_{0} & \text { in } \Omega
\end{array}
$$

where $u_{\mathrm{e}}$ and $v$ are the extracellular and transmembrane potentials, respectively, and $\hat{q}=\left(q_{1}, \ldots, q_{p}\right) \in \mathbb{R}^{p}$ 
represents the vector whose components are the $p$ gating variables, describing the kinetics of the membrane ionic channels; $T>0, \Omega$ is a bounded and open subset of $\mathbb{R}^{3}$ and $\mathbf{n}$ is the outward unit normal vector to $\partial \Omega ; \mathbf{M}_{\mathrm{i}}, \quad \mathbf{M}_{\mathrm{e}}$ and $\mathbf{M}=\mathbf{M}_{\mathrm{i}}+\mathbf{M}_{\mathrm{e}}$ are the conductivity tensors for the intracellular, extracellular and total media, which take into account the effect of cardiac muscle anisotropic fiber structure and of the anisotropic conduction on the macroscopic current flow. Moreover, $C_{\mathrm{m}}$ is the surface capacitance of the membrane, $\chi$ is the ratio of the membrane area per unit of tissue volume, $I_{\text {app }}$ is the stimulation current applied to the intracellular space and $\hat{f}: \mathbb{R} \times \mathbb{R}^{p} \rightarrow \mathbb{R}^{p}$ is a nonlinear vector function.

$I_{\text {ion }}: \mathbb{R} \times \mathbb{R}^{p} \rightarrow \mathbb{R}$ is the ionic current per unit area, carried by the flow of ions across the membrane. Since, we are interested only in the activation phase of action potential, we use Ebihara-Johnson membrane model (EJ) [9]. Nevertheless, numerical simulations of more complex membrane models, such as Beeler-Reuter-EbiharaJohnson (BREJ) $[8,29]$ or others, give substantially the same results as regards the depolarization phase. On the other hand, more recent models such as the one by Luo and Rudy $[20,21]$ improve Beeler-Reuter model by introducing the EJ fast sodium component for the depolarization phase with only minor modifications to parameters calibration and incorporating a slow inactivation gate to represent slow recovery of the sodium channel during the repolarization phase of an action potential. Therefore, in our simulations, $p=2, q_{1}=m, q_{2}=h$ and $I_{\text {ion }}$ is given by

$$
I_{\text {ion }}(v, m, h)=\bar{g}_{\mathrm{Na}} m^{3} h\left(v-v_{\mathrm{Na}}\right)+\bar{g}_{L}\left(v-v_{L}\right),
$$

where $\bar{g}_{\mathrm{Na}}$ is the maximum sodium conductance, $\bar{g}_{L}$ is the specific conductance for repolarization currents, which is assumed to be equal to membrane conductance at rest; $v_{\mathrm{Na}}$ is the equilibrium potential for sodium current and $v_{L}$ is the equilibrium potential for repolarization currents, which coincides with resting potential $v_{\mathrm{r}}$. The ionic current model (2), that will be indicated as EJ in the following, is the sum of two terms: the first one represents sodium current $I_{\mathrm{Na}}$ as described by Ebihara and Johnson [9]. The second one is a linear approximation of repolarization ionic currents (potassium, leakage and others) [36].

The nonlinear term $\hat{f}(v, \hat{q})$ in the gating equations for $m$ and $h$ is of the form

$$
\begin{aligned}
& \hat{f}=\left(f_{1}, f_{2}\right) \\
& f_{i}=f_{i}\left(v, q_{i}\right)=-\left(\alpha_{q_{i}}(v)+\beta_{q_{i}}(v)\right) q_{i}+\alpha_{q_{i}}(v) \\
& i=1,2
\end{aligned}
$$

where $\alpha_{q_{i}}(v)$ and $\beta_{q_{i}}(v)$ are non linear functions of the transmembrane potential as given in Ref. [9].

The Ebihara-Johnson model does not allow to modify extracellular potassium concentration; hence, numerical simulation of a non excitable tissue can be performed by inactivation of fast sodium channels, namely by a reduction of $\mathrm{Na}^{+}$conductance [16], and by a shift of resting potential $v_{\mathrm{r}}$ toward more positive values [34]. The loss of resting potential reduces the maximum upstroke velocity of the action potential $\left(\dot{v}_{\max }\right)$ of a cell and alters $I_{\mathrm{Na}}$ kinetics. At about $v_{\mathrm{r}}=-50 \mathrm{mV}$ membrane excitability is lost and, consequently, total propagation block is present. Sodium conductance reduction can indeed result from inactivation of sodium channels, since the inward sodium current intensity depends on the fraction of open sodium channels when the cell undergoes excitation and on the size of the $\mathrm{Na}^{+}$electrochemical potential gradient (concentration of $\mathrm{Na}^{+}$outside cell, in the extracellular space, relative to $\mathrm{Na}^{+}$concentration inside cell). More precisely, in our numerical simulations of pathological tissue the sodium membrane conductance is reduced to about $37 \%$ of its normal value. Moreover, $\mathrm{K}^{+}$-induced membrane depolarization is obtained by raising the potential value $v_{\mathrm{r}}$ at rest in the injured region $[27,34]$. The parameter calibration was carefully chosen on the basis of the literature.

Following Keener [17], numerical simulations have been performed in rectangular plane sections of a 3D myocardial wall. In this way, we are still able to analyze excitation pathways around obstacles of damaged tissue inside the ventricular wall. Most of the simulations refer to a slab with parallel fibers (see Fig. 1), but the effects of fiber rotation can be taken into account by introducing the tensors

$$
\mathbf{M}_{\mathrm{i}, \mathrm{e}}=\left(\begin{array}{cc}
\sigma^{\mathrm{i}, \mathrm{e}} c^{2}+\sigma_{t}^{\mathrm{i}, \mathrm{e}} & 0 \\
0 & \sigma_{t}^{\mathrm{i}, \mathrm{e}}
\end{array}\right),
$$

where $\sigma_{l}^{\mathrm{i}, \mathrm{e}}$ and $\sigma_{t}^{\mathrm{i}, \mathrm{e}}$ are the conductivity coefficients along and across fibers, respectively, $\sigma^{\mathrm{i}, \mathrm{e}}=\sigma_{l}^{\mathrm{i}, \mathrm{e}}-\sigma_{t}^{\mathrm{i}, \mathrm{e}}, c=$ $\cos \varphi, s=\sin \varphi$ and $\varphi=\varphi(z)$ is the angle between $x$ axis and the unit vector $\mathbf{a}_{l}=\mathbf{a}_{l}(z)$ defining fiber direction at level $z$. In this last case, moving from epicardium to endocardium fiber direction rotates according to the low $\varphi=\varphi(z)$ in planes perpendicular to $z$. Obviously, in Fig. 1 we have $\varphi \equiv 0$; on the other side, when $\varphi$ varies with $z$, the slab under consideration contains the traces of the fibers lying over planes perpendicular to the $z$ axis.

This model neglects the fiber layer curvature and the fiber rotational structure is simplified; nevertheless, the model incorporates the main structural and functional macroscopic characteristics of a portion of ventricular tissue during the excitation process. Therefore, the numerical approximation of this simplified model allows studying the influence of fibers rotation and of anisotropic and inhomogeneous conduction on excitation process. Fiber direction rotates with depth and the combined effect of anisotropic conduction and of fiber rotation is identified as rotational anisotropy.

It has been known for many decades that myocardial necrosis or mechanical injury leads to uncoupling of damaged cells from normal cells. An increase of gap junctional resistance induces electrical cell-to-cell 

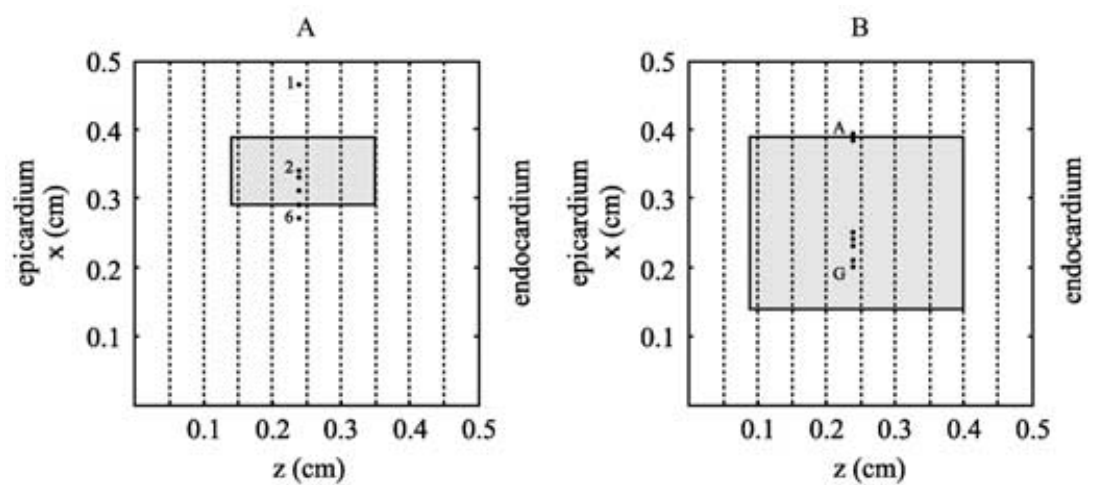

FIGURE 1 Scheme of the domain used in the simulations. The vertical dotted lines represent fiber direction in all the simulations except Fig. 8, where fibers rotate with $z$. The nodes of the uniform grid marked by a dot are labelled from top to bottom, from 1 to 6 (Panel A) and from A to G (Panel B).

uncoupling and ultimately conduction block. The coupling threshold for induction of propagation block in the presence of normal cellular excitability is very high [30]; at decreased membrane excitability (as present in myocardial ischemia), an already moderate decrease in electrical coupling is expected to contribute to propagation slowing and block [30]. Although a continuous bidomain model of propagation does not incorporate a microscopic description of gap junctional resistance, cellular uncoupling can be simulated at a macroscopic level by inserting regions of cardiac tissue with greater effective intracellular resistivity. Therefore, in our simulations of infarcted areas we modified the effective conductivity tensor $\mathbf{M}_{\mathrm{i}}$ : the conductivity coefficients of the inner region are reduced to $5 \%$ of the normal values. Although in some cases osmotic decrease of extracellular space may contribute to cellular potassium loss and may affect extracellular conductivities, in our simulations we neglect such effects and modify only the intracellular coefficients in the pathological region; indeed, in the inner pathological region intracellular conductivities must be reduced, since they are the macroscopic expression of the cellular junctions depauperation, especially in the transverse direction.

All the simulations performed in this work refer to a square section (Fig. 1), with a rectangular subregion of "pathological" tissue embedded in the middle having altered domain properties. These alterations involve both cell-to-cell coupling and membrane excitability as specified above.

\section{NUMERICAL APPROXIMATION}

An integration method based on semidiscrete Galerkin approximation of the R-D system and Crank-Nicholson time-stepping is considered [33]. This method allows to reduce considerably the overall computation time required for solving Hodgkin-Huxley-type models. More precisely, system (1) is approximated in space by means of finite element Galerkin method [28], with piecewise linear, local support polynomials, yielding a differential-algebraic system of the form

$$
\begin{aligned}
& \chi C_{\mathrm{m}} \frac{\mathrm{d} \mathbf{v}}{\mathrm{d} t}(t)+\mathbf{P}^{-1} \mathbf{A}_{i} \mathbf{v}(t)+\mathbf{P}^{-1} \mathbf{i}(\mathbf{v}(t), \mathbf{q}(t)) \\
& =-\mathbf{P}^{-1} \mathbf{A}_{i} \mathbf{u}(t)+\mathbf{P}^{-1} \mathbf{i}_{a}(t) \\
& \frac{\mathrm{d} \mathbf{q}}{\mathrm{d} t}(t)+\mathbf{f}(\mathbf{v}(\mathrm{t}), \mathbf{q}(\mathrm{t}))=0 \quad \mathbf{A u}(t)+\mathbf{A}_{i} \mathbf{v}(\mathrm{t})=\mathbf{0} \\
& \mathbf{q}(0)=\mathbf{q}_{0}=\left(\mathbf{q}_{1,0}, \mathbf{q}_{2,0}\right) \quad \mathbf{v}(0)=\mathbf{v}_{0}
\end{aligned}
$$

where $\quad \mathbf{v}(t)=\left(v_{1}(t), \ldots, v_{N_{h}}(t)\right)^{T} \in \mathbb{R}^{N_{h}}, \quad \mathbf{u}(t)=$ $\left(u_{1}(t), \ldots, u_{N_{h}}(t)\right)^{T} \in \mathbb{R}^{N_{h}} \quad$ and $\quad \mathbf{q}(t)=\left(\mathbf{q}_{1}(t), \mathbf{q}_{2}(t)\right)^{T} \in$ $\mathbb{R}^{2 N_{h}} \forall t \in[0, T]$ are the vectors whose components give an approximation at time $t$ of the nodal values of the solutions $v(t), u_{\mathrm{e}}(t), q_{i}(t), i=1,2$ over an uniform grid. The mass matrix $\mathbf{P}=\left(P_{k, j}\right)$ is definite positive of dimension $N_{h}$, with $P_{k, j}=\int_{\Omega} \varphi_{k} \varphi_{j} \mathrm{~d} \mathbf{x}, k, j=1, \ldots, N_{h}$, $\varphi_{r}, r=1, \ldots, N_{h}$ being the so called shape functions; $\mathbf{A}_{(i)}=\left(a_{r j}^{(i)}\right), a_{r j}^{(i)}=\int_{\Omega}\left(\nabla \varphi_{j}\right)^{T} \mathbf{M}_{(i)} \nabla \varphi_{r} \mathrm{~d} \mathbf{x}, r, j=1, \ldots, N_{h}$, are the symmetric semidefinite positive matrices of order $N_{h} \times N_{h}$ associated to the discretization of the elliptic operators. Finally, $\mathbf{i}_{a}(t)=\left(\int_{\Omega} I_{\text {app }}(t) \varphi_{k} \mathrm{~d} \mathbf{x} ; k=1, \ldots, N_{h}\right)^{T}$ represents the discretization of the stimulus. The integrals are computed by means of the approximated trapezoidal rule (mass lumping). System (3) is discretized by means of Cranck-Nicholson method [24]. This yields the nonlinear finite difference system

$$
\begin{aligned}
\frac{2 \chi C_{\mathrm{m}}}{\Delta t}\left(\mathbf{v}^{l+1}-\mathbf{v}^{l}\right)= & -\mathbf{P}^{-1} \mathbf{A}_{i} \mathbf{v}^{l+1}-\mathbf{P}^{-1} \mathbf{A}_{i} \mathbf{v}^{l} \\
& -\mathbf{P}^{-1} \mathbf{i}\left(\mathbf{v}^{l+1}, \mathbf{m}^{l+1}, \mathbf{h}^{l+1}\right) \\
& -\mathbf{P}^{-1} \mathbf{i}\left(\mathbf{v}^{l}, \mathbf{m}^{l}, \mathbf{h}^{l}\right)-\mathbf{P}^{-1} \mathbf{A}_{i} \mathbf{u}^{l+1} \\
& -\mathbf{P}^{-1} \mathbf{A}_{i} \mathbf{u}^{l}+\mathbf{P}^{-1}\left(\mathbf{i}_{a}\left(t^{l}\right)+\mathbf{i}_{a}\left(t^{l+1}\right)\right) \\
\frac{2}{\Delta t}\left(\mathbf{q}^{l+1}-\mathbf{q}^{l}\right)= & -\mathbf{f}\left(\mathbf{v}^{l+1}, \mathbf{q}^{l+1}\right)-\mathbf{f}\left(\mathbf{v}^{l}, \mathbf{q}^{l}\right)+\mathbf{q}^{l} \\
\mathbf{A} \mathbf{u}^{l+1}+\mathbf{A}_{i} \mathbf{v}^{l+1}= & \mathbf{0}, \quad l=0,1, \ldots, M-1 .
\end{aligned}
$$


The nonlinear terms $\mathbf{i}\left(\mathbf{v}^{l+1}, \mathbf{q}^{l+1}\right)$ and $\mathbf{f}\left(\mathbf{v}^{l+1}, \mathbf{q}^{l+1}\right)$ are then linearized by means of the second order Taylor expansion

$$
\phi\left(y^{l+1}\right)=\phi\left(y^{l}\right)+\phi_{y}\left(y^{l}\right)\left(y^{l+1}-y^{l}\right) .
$$

The overall time discretization method turns out to be second order accurate [33] and yields the linear algebraic system

$$
\begin{gathered}
\left(\frac{2 \chi C_{\mathrm{m}}}{\Delta t} \mathbf{I}+\mathbf{P}^{-1} \mathbf{A}_{i}+\mathbf{J}^{l}\right) \mathbf{v}^{l+1}+\mathbf{P}^{-1} \mathbf{A}_{i} \mathbf{u}^{l+1} \\
+\mathbf{D}_{1}^{l} \mathbf{m}^{l+1}+\mathbf{D}_{2}^{l} \mathbf{h}^{l+1}=\mathbf{b}_{1}^{l} \\
-\frac{\Delta t}{2} \mathbf{D}_{3}^{l} \mathbf{v}^{l+1}+\left(\mathbf{I}+\frac{\Delta t}{2} \mathbf{D}_{4}^{l}\right) \mathbf{m}^{l+1} \\
=-\frac{\Delta t}{2} \mathbf{D}_{3}^{l} \mathbf{v}^{l}+\left(\mathbf{I}+\frac{\Delta t}{2} \mathbf{D}_{4}^{l}\right) \mathbf{m}^{l}+\Delta t \mathbf{b}_{2}^{l} \\
-\frac{\Delta t}{2} \mathbf{D}_{5}^{l} \mathbf{v}^{l+1}+\left(\mathbf{I}+\frac{\Delta t}{2} \mathbf{D}_{6}^{l}\right) \mathbf{h}^{l+1} \\
=-\frac{\Delta t}{2} \mathbf{D}_{5}^{l} \mathbf{v}^{l}+\left(\mathbf{I}+\frac{\Delta t}{2} \mathbf{D}_{6}^{l}\right) \mathbf{h}^{l}+\Delta t \mathbf{b}_{3}^{l} \\
\mathbf{A}_{i} \mathbf{v}^{l+1}+\mathbf{A} \mathbf{u}^{l+1}=\mathbf{0},
\end{gathered}
$$

where, for $k=1, \ldots, N_{h}$,

$$
\begin{gathered}
\mathbf{J}^{l}=\operatorname{diag}\left(J^{l}(k)\right), \quad J^{l}(k)=\chi\left(\bar{g}_{\mathrm{Na}}\left(m_{k}^{l}\right)^{3} h_{k}^{l}+\bar{g}_{L}\right), \\
\mathbf{D}_{i}^{l}=\operatorname{diag}\left(D_{i}^{l}(k)\right), \quad i=1, \ldots, 6, \\
D_{1}^{l}(k)=3 \chi \bar{g}_{\mathrm{Na}}\left(m_{k}^{l}\right)^{2} h_{k}^{l}\left(v_{k}^{l}-v_{\mathrm{Na}}\right), \\
D_{2}^{l}(k)=\chi \bar{g}_{\mathrm{Na}}\left(m_{k}^{l}\right)^{3}\left(v_{k}^{l}-v_{\mathrm{Na}}\right), \\
D_{3}^{l}(k)=\left(1-m_{k}^{l}\right) \frac{\partial \alpha_{m}\left(v_{k}^{l}\right)}{\partial v_{k}^{l}}-\frac{\partial \beta_{m}\left(v_{k}^{l}\right)}{\partial v_{k}^{l}} m_{k}^{l}, \\
D_{4}^{l}(k)=\alpha_{m}\left(v_{k}^{l}\right)+\beta_{m}\left(v_{k}^{l}\right) \\
D_{5}^{l}(k)=\left(1-h_{k}^{l}\right) \frac{\partial \alpha_{h}\left(v_{k}^{l}\right)}{\partial v_{k}^{l}}-\frac{\partial \beta_{h}\left(v_{k}^{l}\right)}{\partial v_{k}^{l}} h_{k}^{l}, \\
D_{6}^{l}(k)=\alpha_{h}\left(v_{k}^{l}\right)+\beta_{h}\left(v_{k}^{l}\right) ; \\
\mathbf{b}_{1}^{l}=\left(\frac{2 \chi C_{\mathrm{m}}}{\Delta t} \mathbf{I}-\mathbf{P}^{-1} \mathbf{A}_{i}+\mathbf{J}^{l}\right) \mathbf{v}^{l}-\mathbf{P}^{-1} \mathbf{A}_{i} \mathbf{u}^{l}+\mathbf{D}_{1}^{l} \mathbf{m}^{l} \\
\left.+\mathbf{D}_{2}^{l} \mathbf{h}^{l}-2 \mathbf{P}^{-1} \mathbf{i}\left(\mathbf{v}^{l}, \mathbf{q}^{l}\right)+\mathbf{P}^{-1} \mathbf{i}_{a}\left(t^{l+1}\right)+\mathbf{P}^{-1} \mathbf{i}_{a}\left(t^{l}\right)\right) \\
\mathbf{b}_{2}^{l}=\left(\alpha_{m}\left(v_{1}^{l}\right)+\beta_{m}\left(v_{1}^{l}\right)\right) m_{1}^{l}+\alpha_{m}\left(v_{1}^{l}\right), \ldots,-\left(\alpha_{m}\left(v_{N_{h}}^{l}\right)\right. \\
\left.\left.+\beta_{h}\right)\right)^{T},
\end{gathered}
$$

$$
\begin{aligned}
\mathbf{b}_{3}^{l}= & \left(-\left(\alpha_{h}\left(v_{1}^{l}\right)+\beta_{h}\left(v_{1}^{l}\right)\right) h_{1}^{l}+\alpha_{h}\left(v_{1}^{l}\right), \ldots,-\left(\alpha_{h}\left(v_{N_{h}}^{l}\right)\right.\right. \\
& \left.\left.+\beta_{h}\left(v_{N_{h}}^{l}\right)\right) h_{N_{h}}^{l}+\alpha_{h}\left(v_{N_{h}}^{l}\right)\right)^{T} .
\end{aligned}
$$

At every time step $t^{l}$, the totally discretized system can be rewritten in the block form

$$
\begin{aligned}
\mathbf{G}^{l} \mathbf{v}^{l+1}+\mathbf{A}_{i} \mathbf{u}^{l+1} & =\mathbf{E}^{l} \mathbf{v}^{l}-\mathbf{A}_{i} \mathbf{u}^{l}+\mathbf{F}^{l} \\
\mathbf{A}_{i} \mathbf{v}^{l+1}+\mathbf{A} \mathbf{u}^{l+1} & =\mathbf{0},
\end{aligned}
$$

where the gating equations have been incorporated into the terms $\mathbf{G}^{l}, \mathbf{E}^{l}$ and $\mathbf{F}^{l}$. The algebraic system (4) is solved by means of a nested iterative method. The outer iteration is given by a block Gauss-Seidel scheme

$$
\begin{gathered}
\mathbf{v}^{l+1,0}=\mathbf{v}^{l} \quad \mathbf{u}^{l+1,0}=\mathbf{u}^{l} \\
\text { for } j=0,1, \ldots, \nu \quad \mathbf{G}^{l} \mathbf{v}^{l+1, j+1}=-\mathbf{A}_{i} \mathbf{u}^{l+1, j}+\mathbf{g}^{l} \\
\mathbf{A u}^{l+1, j+1}=-\mathbf{A}_{i} \mathbf{v}^{l+1, j+1}
\end{gathered}
$$

with $\mathbf{g}^{l}=\mathbf{E}^{l} \mathbf{v}^{l}-\mathbf{A}_{i} \mathbf{u}^{l}+\mathbf{F}^{l}$. At each step of this outer iteration, we have to solve two linear algebraic systems of order $N_{h}$, for which different linear solvers have been used and compared [33].

The procedure for computing the activation time is the following: at first, the values of $\partial I_{\mathrm{Na}} / \partial t$ are obtained from $I_{\mathrm{Na}}$ by means of a second order centered difference; then the maximum of $\left|\partial I_{\mathrm{Na}} / \partial t\right|$ is localized on an interval containing three successive time instants and a local quadratic interpolant of $\left|\partial I_{\mathrm{Na}} / \partial t\right|$ is built; subsequently the activation time is computed as the instant at which such interpolant is maximum.

All the simulations presented in the following refer to uniform grids over a square section, with a rectangular subregion of "pathological" tissue embedded in the middle (Fig. 1). The position of the depressed area is such to allow the occurrence of uniform propagation. To minimize discretization errors associated with the discontinuity in domain properties at the interface between the "normal" and the "pathological" areas, a thin border region was inserted with domain properties intermediate between those of the two regions. The employed discretization step $\Delta t=0.02 \mathrm{~ms}$ and $\Delta x=\Delta z=0.005 \mathrm{~cm}$ were chosen so that further reduction did not produce any significant variation of the numerical results. The space discretization steps were then doubled in order to analyze the numerical artifacts associated with non accurate numerical approximations of the bidomain model. The relative error between the two different simulations may reach $20 \%$ in the region of normal propagation.

\section{RESULTS}

Several numerical simulations have been performed in order to analyze the effect of changes in cellular coupling 
TABLE I Parameter values for the pathological and intermediate regions, used in the simulations of the action potential in the presence of a subregion with depressed EJ membrane

\begin{tabular}{lllc}
\hline Intermediate & Value & Infarcted & Value \\
\hline$\sigma_{l}^{i}$ & $0.5 \mathrm{mS} / \mathrm{cm}$ & $\sigma_{l}^{i}$ & $0.1 \mathrm{mS} / \mathrm{cm}$ \\
$\sigma_{t}^{i}$ & $0.05 \mathrm{mS} / \mathrm{cm}$ & $\sigma_{t}^{i}$ & $0.01 \mathrm{mS} / \mathrm{cm}^{i}$ \\
$\bar{g}_{\mathrm{Na}}$ & $24 \mathrm{mS} / \mathrm{cm}^{2}$ & $\bar{g}_{\mathrm{Na}}$ & $13 \mathrm{mS} / \mathrm{cm}^{2}$ \\
$v_{r}$ & $-70 \mathrm{mV}$ & $v_{r}$ & $-60 \mathrm{mV}$ \\
\hline
\end{tabular}

and in membrane excitability on propagation velocity and on the associated generation of fractionated electrograms. In order to eliminate other factors that may contribute to the generation of such phenomena, we consider at first a cardiac section with fibers parallel to $x$ axis $(\varphi=0)$. Fiber rotation will be considered later on.

The first group of simulations ("Electrogram fractionation" section) highlights the relation between cellular coupling variations and the appearance of fractionation in the electrograms. Our simulations showed that fractionation occurs in the presence of discontinuities in the conductivity properties of the cardiac tissue, regardless of the membrane model employed. On the contrary, it seems that the membrane current model plays a role in the generation of conduction blocks, since a cell having a decreased membrane excitability requires a greater stimulating current to raise it to threshold and the loading effect of the underlying tissue prevents the excitation of the tissue still at rest. In this regard, it is worth noticing that conduction blocks can actually occur in the simulation of propagation in a bidomain wall of infarcted myocardium, for instance in the presence of excessive inactivation of $I_{\mathrm{Na}}$ or depolarization of $v_{\mathrm{r}}$. Nevertheless, in some cases, in a discretization of continuous macroscopic models like the bidomain, the occurrence of propagation failure has to be considered a numerical artifact. This topic will be treated in "Effects of discretization-Conduction blocks" section, where also other determinants of conduction blocks are considered, such as fiber rotation.

\subsection{Electrogram Fractionation}

The first group of simulations refers to a section of dimension $0.5 \mathrm{~cm} \times 0.5 \mathrm{~cm}$ (Fig. 1), where fibers are parallel to $x$ axis and an uniform stimulation is applied along $z$ axis to simulate plane wave propagation along fibers, with intensity $i_{\text {app }}=I_{\text {app }} / \chi=1.44 \mathrm{~mA} / \mathrm{cm}^{2}$. The conductivity values along $x$ and $z$ direction and the sodium conductance value for the pathological and intermediate regions are listed in Table I; the remaining parameters coincide with those of Table II. Propagation is relatively fast in $x$ direction and slow in $z$ direction, because of anisotropic conductivities.

Numerical simulations show that the inward sodium current reduction in the pathological area yields a reduction of the rate $(\mathrm{d} v / \mathrm{d} t)$ and amplitude of depolarization, of conduction velocity, of the electrotonic current flux, of $I_{\mathrm{Na}}$ current and of extracellular potential amplitude. In some cases, further depolarization and inactivation of the $\mathrm{Na}^{+}$channels may decrease excitability of cardiac fibers to such an extent that it may also generate conduction blocks. In fact, the fraction of $\mathrm{Na}^{+}$channels available for opening is determined largely by the level of membrane potential at which an action potential is initiated. For cardiac cells with persistently low levels of resting potentials (which may be between -60 and $-70 \mathrm{mV}$ ) caused by disease, a significant fraction of the $\mathrm{Na}^{+}$channels is inactivated and, therefore, unavailable for activation by a depolarizing stimulus. Conduction may be blocked in regions where cells are depolarized at about $v_{\mathrm{r}}=-50 \mathrm{mV}$. Thus, in a diseased region, there may be some areas of slow conduction and some areas of conduction blocks, possibly depending on the level of resting potential and on the corresponding degree of inactivation of sodium channels. This combination may cause reentry of excitation.

Another factor that may cause slow conduction and blocks necessary for reentry is an increase of gap junctional resistance which induces electrical cell-to-cell uncoupling, leading to a reduced spread and magnitude of the side-to-side current along the myocardial fibers [14].

Figure 2 shows the transmembrane potential (A), the sodium current (B), the extracellular potential (C) waveforms at the nodes selected in Fig. 1, Panel A and the isochrone level lines of the activation time (D). The discretization steps employed are $\Delta x=\Delta z=0.005 \mathrm{~cm}$ and $\Delta t=0.02 \mathrm{~ms}$.

Looking at the different waveforms and at the sodium current profiles, we see evident differences from one node to another. In the presence of such heterogeneous signals, it is sometimes difficult to differentiate true propagating electrical activity (local cardiac activation) from electrotonic depolarization. Uniform propagation occurs

TABLE II Parameters values in normal tissue

\begin{tabular}{lc}
\hline Parameter & Value \\
\hline$i_{\text {app }}$ current & $1.44 \mathrm{~mA} / \mathrm{cm}^{2}$ \\
$i_{\text {app }}$ duration & $0.5 \mathrm{~ms}$ \\
$\chi$ & $2000 \mathrm{~cm}^{-1}$ \\
$v_{\mathrm{Na}}$ & $33.4 \mathrm{mV}$ \\
$\bar{g}_{\mathrm{L}}$ & $0.05 \mathrm{mS} / \mathrm{cm}^{2}$ \\
$C_{\mathrm{m}}$ & $1.0 \mu \mathrm{NF} / \mathrm{cm}^{2}$ \\
$\sigma_{l}^{i}$ & $2.0 \mathrm{mS} / \mathrm{cm}^{i}$ \\
$\sigma_{t}^{j}$ & $0.2 \mathrm{mS} / \mathrm{cm}^{j}$ \\
$\sigma_{l}^{\mathrm{e}}$ & $8.0 \mathrm{mS} / \mathrm{cm}^{\mathrm{e}}$ \\
$\sigma_{t}^{\mathrm{e}}$ & $2.0 \mathrm{mS} / \mathrm{cm}^{2}$ \\
$\overline{g_{\mathrm{Na}}}$ & $35.0 \mathrm{mS} / \mathrm{cm}^{2}$ \\
$v_{r}$ & $-80 \mathrm{mV}$ \\
\hline
\end{tabular}



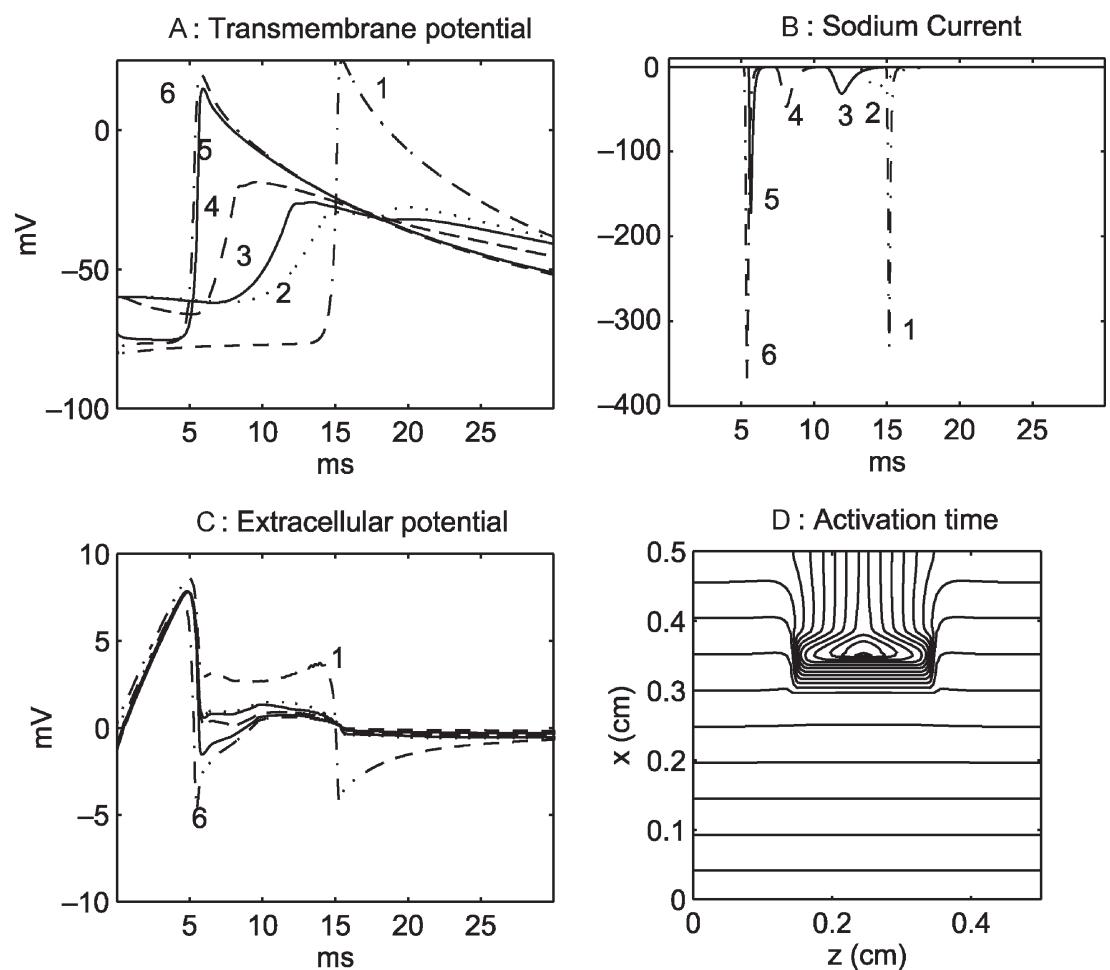

FIGURE 2 Time course of $v$ (Panel A), $I_{\mathrm{Na}}\left(\right.$ Panel B) and $u_{\mathrm{e}}$ (Panel C) at the nodes whose locations are marked in Fig. 1, Panel A. The transmembrane potential waveforms are numbered with the index of the node they refer to. $I_{\mathrm{Na}}$ is expressed in $\mu \mathrm{A} / \mathrm{cm}^{2}$. Panel D displays isochrone level lines of activation time with a time step of $1 \mathrm{~ms}$, computed as the instant of maximum $\left|\partial I_{\mathrm{Na}} / \partial t\right|$. A line stimulus is applied at an edge along the $z$ axis, with a duration of $0.5 \mathrm{~ms}$ to initiate propagation. Discretization steps: $\Delta x=\Delta z=0.005 \mathrm{~cm}$ and $\Delta t=0.02 \mathrm{~ms}$.

if a patch of membrane can supply enough current to depolarize itself and charge the capacitance of the neighboring tissue, which has yet to depolarize. Otherwise current flow is no longer regenerative, only an electrotonic deflection appears and conduction will eventually terminate. In [40] Witkowski et al. proposed the presence of a balanced inwardly and outwardly directed transmembrane charge, obtained from the ratio of the inward $\left(A_{-}\right)$to outward $\left(A_{+}\right)$area under the cardiac transmembrane current curve $\left(A_{-}\left(I_{\mathrm{m}}\right) / A_{+}\left(I_{\mathrm{m}}\right)\right.$, where $\left.I_{\mathrm{m}}=C_{\mathrm{m}} v_{\mathrm{t}}+I_{\text {ion }}\right)$, as a criterion to differentiate propagating from electrotonic deflections. They identified the value $A_{-}\left(I_{\mathrm{m}}\right) / A_{+}\left(I_{\mathrm{m}}\right) \approx 1$ as an index of propagating activity and the value $A_{-}\left(I_{\mathrm{m}}\right) / A_{+}\left(I_{\mathrm{m}}\right) \approx 0$ as corresponding to electrotonic deflection. The values in between these two extremes $\left(0.0<A_{-}\left(I_{\mathrm{m}}\right) / A_{+}\left(I_{\mathrm{m}}\right)<0.9\right)$ were termed transitional and correspond to a depressed activation. Under these conditions, we cannot be certain if the observed signals represent high-level electrotonic interactions versus some level of decrementing conduction. The area under the cardiac transmembrane current curve was computed using the iterated trapezoidal quadrature rule. Another possible location-dependent quantitative measure of conduction is the safety factor of Wang and Rudy [39]. In this work, we assume the value $A_{-}\left(I_{\mathrm{m}}\right) / A_{+}\left(I_{\mathrm{m}}\right)=0.2$ as the threshold value for discriminating between true local cardiac activation and electrotonic depolarization. When such a threshold value is not reached, the membrane patch is considered inactive and the mesh point under consideration is regarded as inexcitable; at this node the activation time selected as maximizing $\partial I_{\mathrm{Na}} / \partial t$ is not significant and is ignored when tracing the activation isochrones.

As a consequence of fiber orientation, depolarization proceeds from bottom to the top, parallel to $x$ axis and enters the pathological region from below and following a diagonal path. In this region, we record a severely depressed conduction velocity, but the ratio of negative to positive area under the $I_{\mathrm{m}}$ curves attests to the presence of true local propagated activation over the entire volume.

When an action potential propagates at a constant velocity, a biphasic electrogram is usually recorded by a unipolar electrode near the surface of the tissue; on the contrary, when the action potential passes through regions with discontinuous membrane properties and heterogeneous distribution of cell-to-cell coupling resistance the corresponding electrograms deviate from normal and may present several deflections, occurring at the times when the activation wavefront crosses the discontinuity barriers [23].

In Fig. 2, Panel $\mathrm{C}$, we can notice the presence of fractionated electrograms, occurring when the wavefront crosses the boundary of the region having reduced conductivity. The potential waveforms at some of the nodes from 1 to 6 are plotted separately in Fig. 3, in order to better show this phenomenon. While node 6 is characterized by a biphasic extracellular potential where the fastest deflection corresponds to local activation, the other 

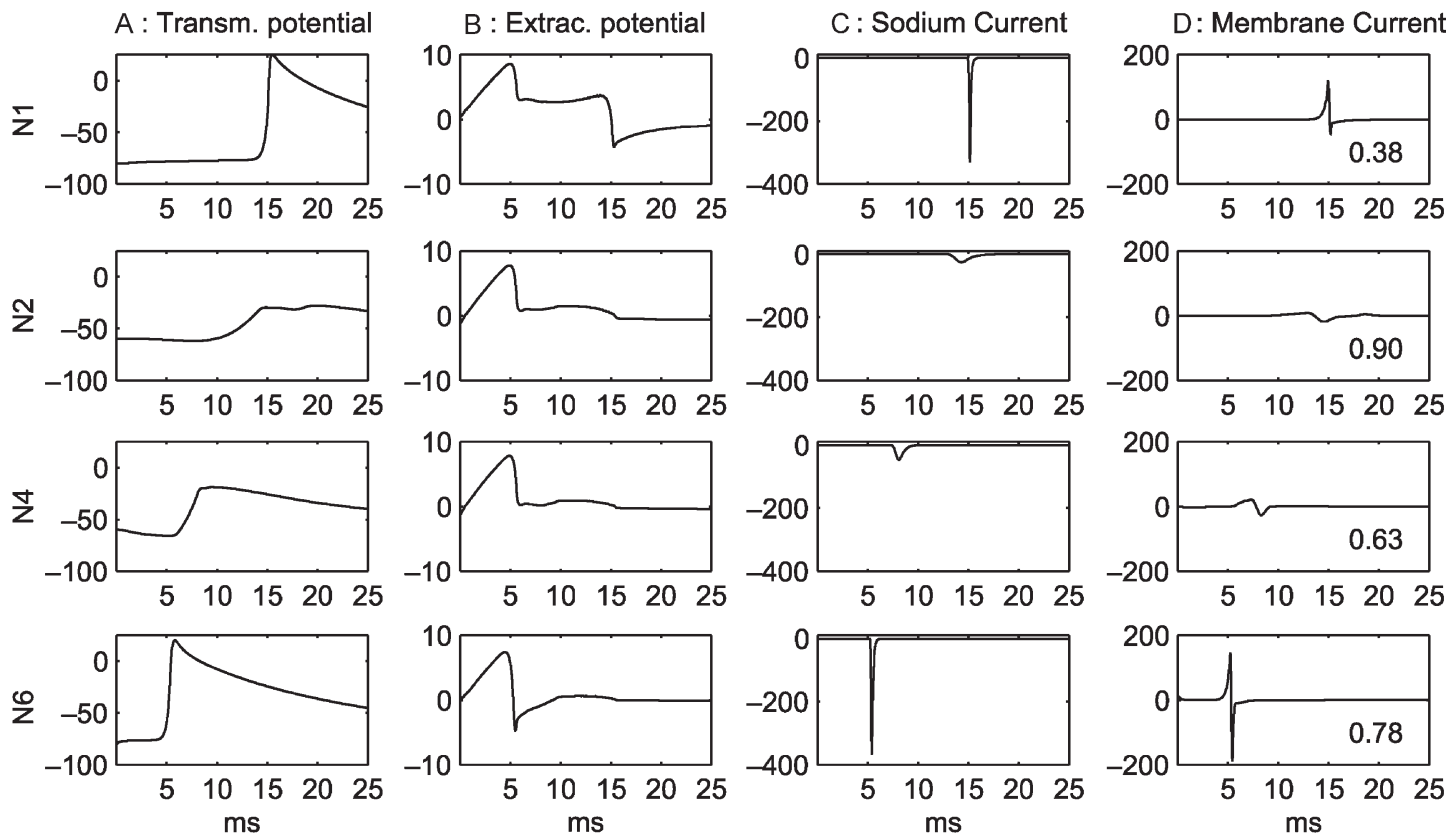

FIGURE 3 Time course of $v$ (Panel A), $u_{\mathrm{e}}$ (Panel B), $I_{\mathrm{Na}}\left(\right.$ Panel C) and $I_{m}$ (Panel D) at the nodes 1, 2, 4 and 6, whose locations are marked in Fig. 1, Panel A. $v$ and $u_{\mathrm{e}}$ are expressed in $\mathrm{mV}$, while $I_{\mathrm{Na}}$ and $I_{m}$ in $\mu \mathrm{A} / \mathrm{cm}^{2}$. The ratio of negative to positive area under the $I_{m}$ curves is given in the lower right corner of each box of Panel D. Discretization steps: $\Delta x=\Delta z=0.005 \mathrm{~cm}$ and $\Delta t=0.02 \mathrm{~ms}$.

nodes exhibit polyphasic electrograms with a dominant deflection at about $5-6 \mathrm{~ms}$, which corresponds to the time when the front reaches the slow conducting region and which is not an index of local activation at that node. This deflection represents a passive extracellular current, which does not modify the transmembrane potential and which gives distance (non local) informations on the shape and dimension of the depressed region. At node 1, the second deflection corresponds to the real local activation, as attested by the $I_{\mathrm{Na}}$ and $I_{\mathrm{m}}$ profiles, while at the nodes 2 and 4 the deflection corresponding to local activation, respectively, at 13.74 and $7.8 \mathrm{~ms}$, is little pronounced. Finally, the small deflection occurring at about $16 \mathrm{~ms}$ at nodes 2, 4, 6 corresponds to the time when the wavefront reaches the upper boundary of the depressed region; therefore, the discontinuity in the conductivity properties of the medium affects also nodes like 6, lying in the normal region and which should not present alterations in the waveform profiles. All these results are in agreement with the ones in [23], where a monodomain model of a cardiac sheet imbedded into an unbounded conducting medium is considered versus the present bidomain model of an insulated cardiac slab. The slower the conduction in the inner region, the more this phenomenon is evident. In fact, it is known [36,33] that slow conduction produces extracellular electrograms with smaller amplitude and less steep deflections with respect to the ones occurring during fast propagation. As the propagation velocity of the excitation wavefront in the inner region slows down, the polyphasic nature of electrograms becomes more evident, the deflection amplitude, corresponding to local activation, becomes smaller and less steep. However, it is worth noting that propagation speed reduction is not able to cause electrogram fractionation by its own.

\subsection{Effects of Discretization-Conduction Blocks}

The use of coarse grids in the numerical discretization of the bidomain problem (1), can induce in the approximate solution a behavior which differs from the one predicted by the continuous model; more precisely, the wavefront propagation exhibits features that resemble those of discrete cellular models. In this section, we analyze the effects of discretization in the presence of areas of slow conduction. It should be remarked that, since in our case the discrete model derives from the approximation of a continuous bidomain model, any behavior, which is not consistent with the continuous theory can only be due to numerical artifact, and cannot be viewed as an improvement on continuous theory. Nevertheless, being aware about the differences that can occur using coarse rather than fine grids, can be useful for two reasons:

1. it warns against misunderstandings and wrong interpretations of numerical results;

2. it may give informations and hints about possible different results that can be obtained using discrete cellular models of cardiac tissue.

The simulation of Figs. 2 and 3 has been repeated using a coarse space mesh with discretization steps $\Delta x=$ $\Delta z=0.01 \mathrm{~cm}$ and $\Delta t=0.02 \mathrm{~ms}$ and is displayed in Figs. 4 and 5. In Fig. 4, we can see high frequency oscillations in the fractionated extracellular potential profiles. It is worth 

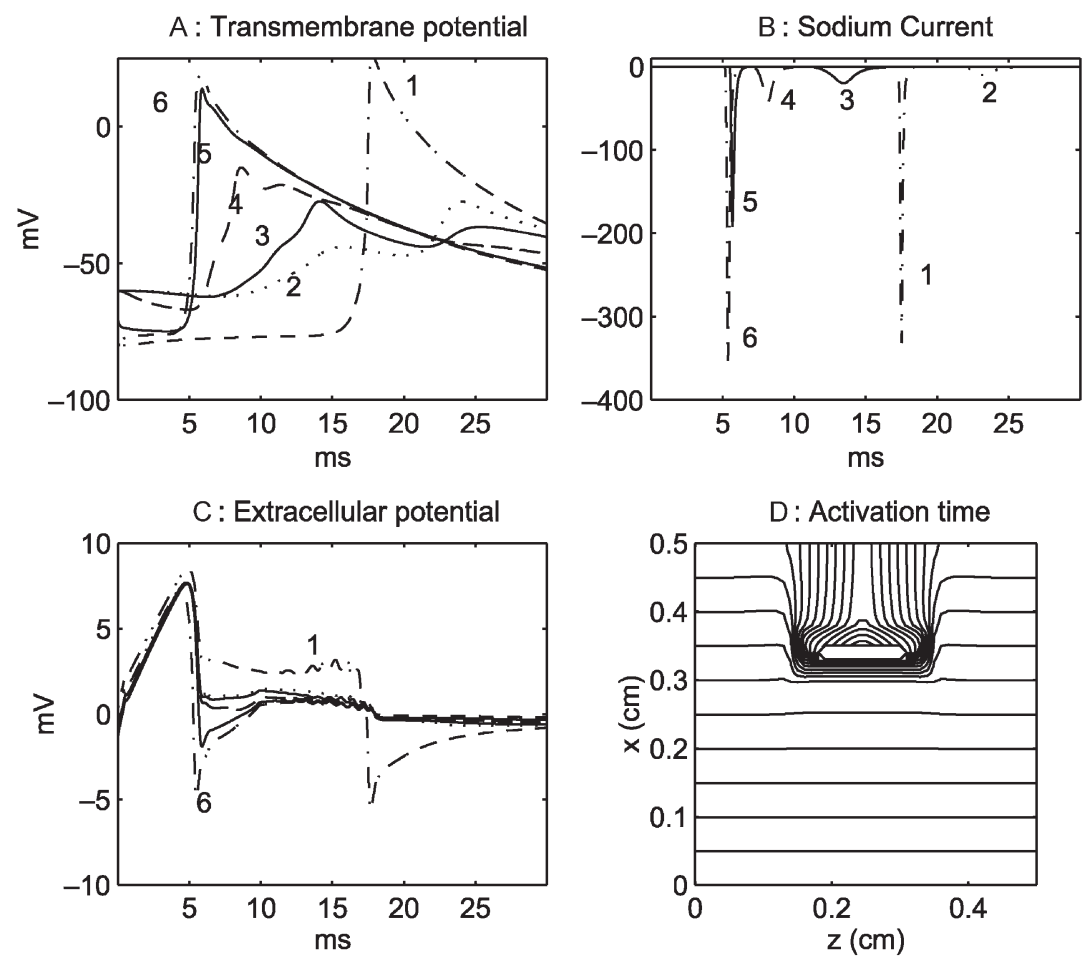

FIGURE 4 Same format as in Fig. 2 but using a coarse mesh with steps: $\Delta x=\Delta z=0.01 \mathrm{~cm}$ and $\Delta t=0.02 \mathrm{~ms}$.

noting that they have no physiological meaning, but merely a numerical origin. They appear at times when the wavefront propagates slowly in the injured region. In fact, the wavefront conduction velocity varies from a maximum of $0.0519 \mathrm{~cm} / \mathrm{ms}$ at nodes in the normal region to a minimum of $0.0016 \mathrm{~cm} / \mathrm{ms}$ and the waveform oscillations occur during the time interval when the wavefront crosses the slow conduction region. As we can see from Figs. 2 and 3, these spurious oscillations can be reduced and completely suppressed by reducing the space mesh. On the contrary, no improvement in the quality of waveforms derives from a reduction of $\Delta t$ only.

A very interesting aspect of Fig. 4 is the presence of a considerable slackening of conduction speed in the
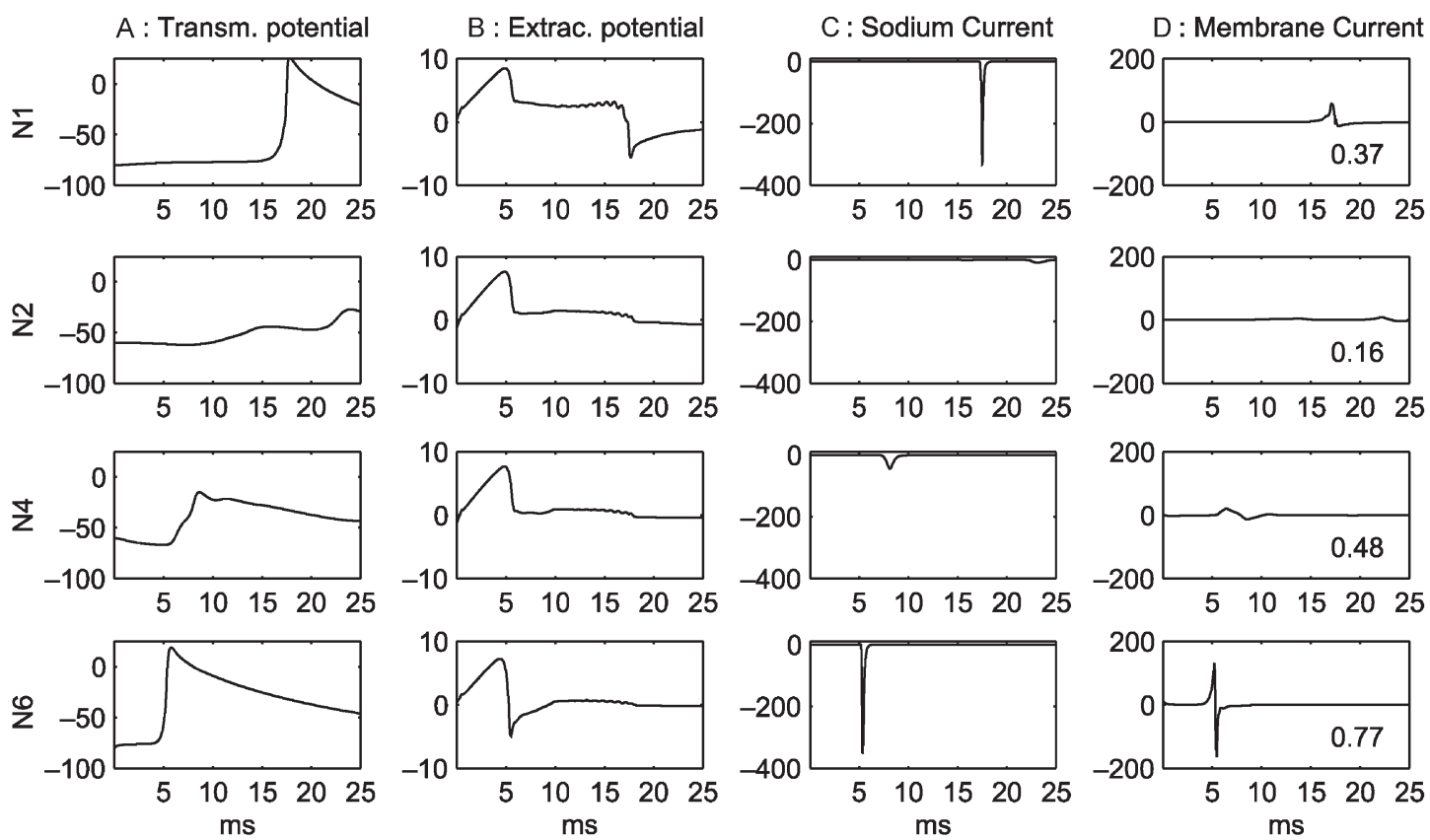

FIGURE 5 Same format as in Fig. 3 but using a coarse mesh with steps: $\Delta x=\Delta z=0.01 \mathrm{~cm}$ and $\Delta t=0.02 \mathrm{~ms}$. 
longitudinal direction, immediately after the interface with the injured region; the wavefront travels around the obstacle represented by the depressed area and enters it following a lateral path. However, differently from Fig. 2, in this case the local circuit current is unable to propagate through the inner region and it is only sufficient to depolarize passively (i.e. electrotonically) the tissue. Therefore, two depolarization wavefronts enter the damaged area: one from below, having severely depressed conduction velocity, and the other from above, following lateral excitation pathways. The former is responsible for the depolarization of nodes from 6 back to 3 . Conduction is so depressed, that it fails before reaching node 2 (we can see from Fig. 5, Panel D that no local cardiac activation is present at node 2). The latter gives rise to a new depolarization current at the internal nodes, which were not sufficiently excited from below. However, this current cannot activate cellular membrane at the center of the depressed region (Fig. 4, Panel D), since the membrane is not completely recovered at those points (see in particular nodes 2 and 3 ). This can be argued by looking at $I_{\mathrm{Na}}$ course at node 3 , where the only negative $I_{\mathrm{Na}}$ peak corresponds to the front moving from below, while the second depolarization current does not produce any active membrane response, but only an electrotonic deflection.

The simulation of Figs. 4 and 5 has been repeated in the case of depressed EJ membrane with a strongly depressed leakage current. The ionic current in both the normal and the pathological region is of Ebihara and Johnson type, with $g_{L}=0.001 \mathrm{~ms} / \mathrm{cm}^{2}$. The other physiological parameters are the same as in the previous simulations, with
$\Delta x=\Delta z=0.01 \mathrm{~cm}$ and $\Delta t=0.02 \mathrm{~ms}$. Fibers are parallel to $x$ axis and a uniform stimulation is applied along $z$ axis.

Time courses of transmembrane and extracellular potentials are plotted in Fig. 6. At nodes 5 and 6 the largest electrogram deflection corresponds to local activation, while at the other nodes it corresponds to the moment when the front reaches the boundary of the depressed region.

It should be remarked that the absence of leakage current prevents the occurrence of conduction block, even in the presence of large meshes. The wavefront propagates through all the tissue, even if propagation speed varies according to the variation of conductivity coefficients inside the domain. Sodium current intensity at nodes in the injured region is reduced, yet it gives rise to an active response of the tissue. In fact, membrane excitability and the excitation threshold are highly determined by ionic current; if we modify such current term, excitation threshold may change and the same mesh that gave misleading information and blocks before, now can describe propagation more faithfully. Therefore, we can conclude that the formation of numerical blocks is conditioned by many factors, such as ionic current, resting potential value, cell-to-cell coupling, since all these factors influence conduction speed.

Propagation is also conditioned by loading effects of the underlying tissue and by the dimension of the damaged region. Figure 7 refers to an inner depressed region of dimension $0.31 \mathrm{~cm} \times 0.25 \mathrm{~cm}$. Discretization steps are $\Delta x=\Delta z=0.005 \mathrm{~cm}$ and $\Delta t=0.02 \mathrm{~ms}$ and parameters values are listed in Tables I and II. The waveforms are
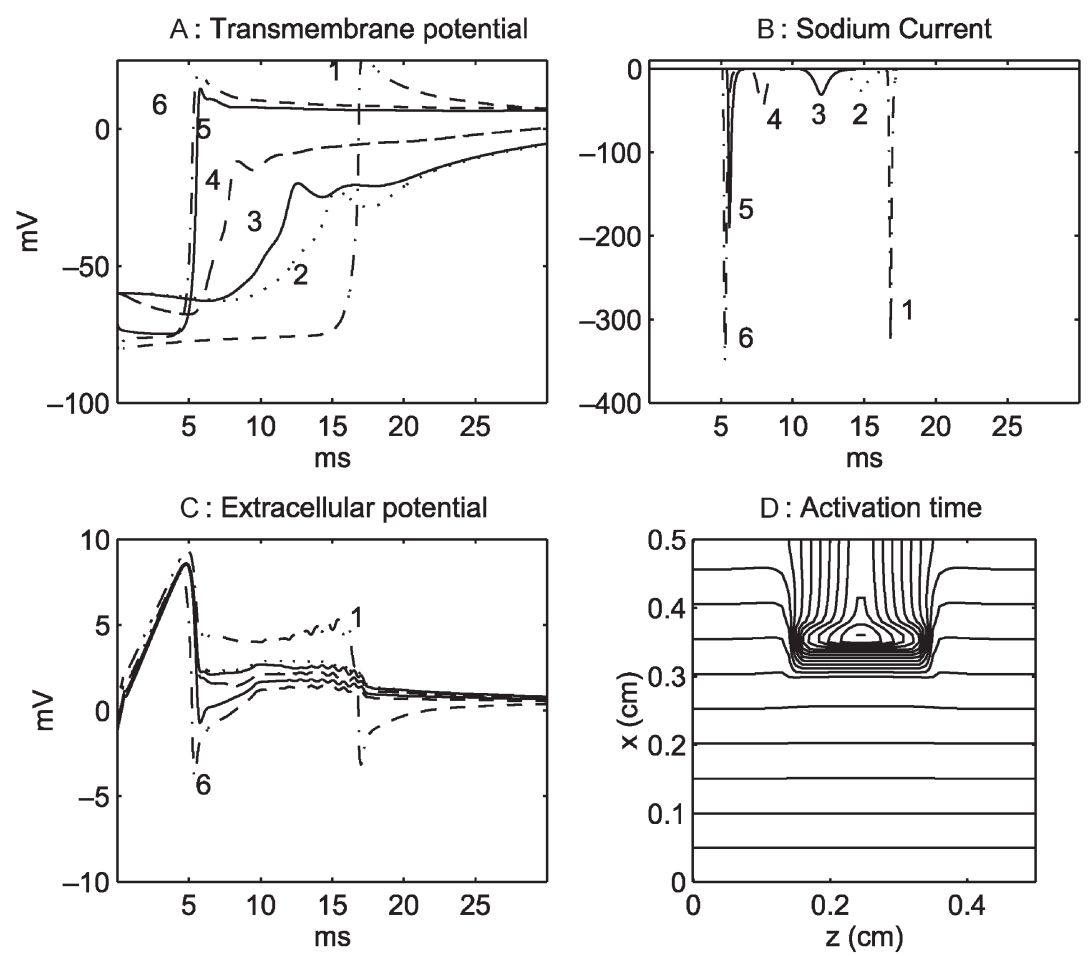

FIGURE 6 EJ membrane with severely depressed leakage current: same format as in Fig. 2. Discretization steps: $\Delta x=\Delta z=0.01 \mathrm{~cm}$ and $\Delta t=$ $0.02 \mathrm{~ms}$ 

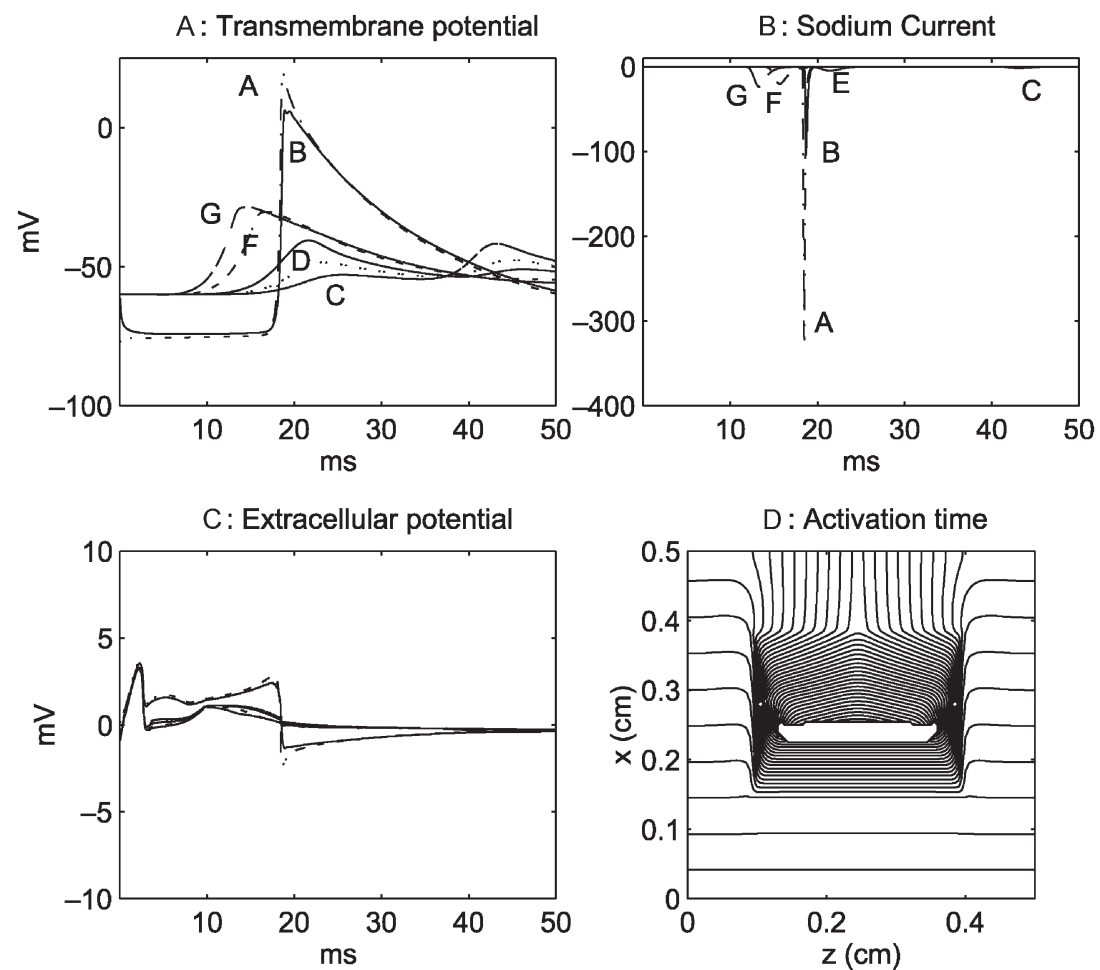

FIGURE 7 Time course of $v$ (Panel A), $I_{\mathrm{Na}}\left(\right.$ Panel B), $u_{\mathrm{e}}$ (Panel C) at the nodes whose locations are marked in Fig. 1, Panel B. The transmembrane potential and sodium current profiles are labelled with the index of the node they refer to. Panel D displays isochrone level lines of activation time with a time step of $1 \mathrm{~ms}$, computed as the instant of maximum $\left|\partial I_{\mathrm{Na}} / \partial t\right|$. A line stimulus is applied at an edge along the $z$ axis, with a duration of $0.5 \mathrm{~ms}$ to initiate propagation. Discretization steps: $\Delta x=\Delta z=0.005 \mathrm{~cm}$ and $\Delta t=0.02 \mathrm{~ms}$.

plotted at nodes A, B, C, D, E, F and G, corresponding to points $(48 \Delta x, j \Delta y), \quad j=79,77,50,48,46,42,40 \quad$ (see Fig. 2, Panel B).

As we can see from the time course of transmembrane potential at nodes $\mathrm{C}, \mathrm{D}$ and $\mathrm{E}$, the wavefront propagating from below is not sufficient to excite tissue inside the depressed region and this generates a conduction block. In particular, at node $\mathrm{D} I_{\mathrm{Na}}$ is identically equal to zero. Subsequently, the wavefront enters the inner region following lateral pathways, proceeding at first across fiber; however, it cannot activate the still partially depolarized tissue, as we can see from the $I_{\mathrm{Na}}$ profile at node C.

Finally, we emphasize that block formation is conditioned also by fibers rotation and from the position of the injured regions with respect to the direction of propagating wavefront [19]. Figure 8 refers to a normal cardiac tissue containing a depressed Ebihara-Johnson subregion according to scheme of Fig. 1, Panel A, with parameters specified in Tables I and II. In this simulation, fibers are supposed to rotate according to the law $\varphi(z)=2 \pi z / 3-\pi / 2$ and a point stimulus is applied near the origin, with a duration of $0.5 \mathrm{~ms}$ to initiate propagation. The activation time is computed as the instant which maximizes $\left|\partial I_{\mathrm{Na}} / \partial t\right|$ and the isochrones are traced on the region recognized as active, i.e. $A_{-}\left(I_{\mathrm{m}}\right) / A_{+}\left(I_{\mathrm{m}}\right)>0.2$. In Panel $\mathrm{D}$, the excitation wavefront propagates towards north-east and travels around the depressed region. Isochrones are distorted from elliptic shape because of fibers orientation; in fact, conductivities in the $x$ and $z$ directions are equal at level $z=0$, since they are both across fiber, and become progressively more different as we reach the endocardium at $z=0.5$. Differently from Fig. 2, we can now observe the formation of a conduction block, since the local circuit current is unable to propagate throughout the inner region and to lead the whole tissue to threshold. In particular, node 2 exhibits a transitional severely depressed activation: from the transmembrane potential profile (Panel A), we observe a first depolarization current (electrotonic deflection), sustained by a second depolarization current, which corresponds to a very small inward sodium current (Panel B). The ratio of negative to positive area under the $I_{\mathrm{m}}$ curve is equal to 0.22 . This discrepancy from Fig. 2 is due to the different angle of interaction of the wavefront with the obstacle. Again we observe the occurrence of electrogram fractionation (Panel C).

\section{DISCUSSION}

The difficulties connected with the numerical simulation of slow propagation have been observed in the monodimensional core-conductor model in $[33,36,38]$. For an action potential propagating along a cilindrical fiber, Spach [36] showed that large space discretization steps yielded numerical results differing from those 
predicted by the theory related to the continuous model. The sensitivity of the numerical results to the choice of space steps in the discretization of the cable equation depends strongly on the value of the propagation velocity and was already observed by Cooley and Dodge [6].

Although conduction blocks can really occur in the presence of uncoupled cells or depressed membrane, yet it is important to underline the fact that the block in Fig. 4 is strictly correlated to space discretization of the continuous model and must be considered as a numerical artifact; in fact, Figs. 2 and 3, obtained using the finer steps $\Delta x=\Delta z=0.005 \mathrm{~cm}$, show a greater propagation speed in the depressed region. This proves that coarse meshes in relation to propagation speed, besides introducing spurious oscillations in the waveforms, can also generate altered conduction speed and conduction blocks. More precisely, while conduction velocity in the outer region is such to allow the use of a $0.01 \mathrm{~cm}$ step, in the inner region propagation becomes so slow as to require the use of smaller space steps in order to describe excitation wavefront propagation faithfully.

It should be excluded that the presence of the oscillations on the electrogram profiles obtained with coarse meshes results from the algorithm used in the simulations to solve the discrete problem, i.e. the nested iterations (5)-(6) for the solution of the final finite difference system. Neither it is due to the singular nature of the R-D system which imposes to select, at each iteration, among the infinitely many solutions $\mathbf{u}$ the one having zero potential average on the cardiac domain. Obviously, if we increase conductivities in the injured region it is possible to eliminate these spurious oscillations, which are a consequence of the coarseness of the mesh with respect to the conduction velocity. Therefore, the simulation of pathological processes and of slow conduction requires the use of spatial refinement techniques in order to avoid artifacts affecting the numerical simulation of the action potential propagation.

In our numerical method, the discretization steps can be chosen in a wide range of values. In fact, the analysis of spectral stability [24] of the numerical method reveals that the stability of the final finite difference system (4) is assured at every time step $t^{l}$ if

$$
C_{1}^{l}(k)+C_{2}^{l}(k)+1>0, \quad k=1, \ldots, N_{h},
$$

where

$$
\begin{gathered}
C_{1}^{l}(k)=\frac{\Delta t^{2}}{4} D_{1}^{l}(k)\left[1+\frac{\Delta t}{2} D_{4}^{l}(k)\right]^{-1} D_{3}^{l}(k), \\
C_{2}^{l}(k)=\frac{\Delta t^{2}}{4} D_{2}^{l}(k)\left[1+\frac{\Delta t}{2} D_{6}^{l}(k)\right]^{-1} D_{5}^{l}(k) .
\end{gathered}
$$

Typically, stability constraints establish a bond between $\Delta t$ and $\Delta x, \Delta z$ such that a decrease in $\Delta x, \Delta z$ yields a decrease in $\Delta t$ to have stable results. Condition (7), where only $\Delta t$ appears, is always verified in our simulations and the time step choice is conditioned mostly by accuracy requirements rather than by stability constraints. On the contrary, the numerical solution is highly conditioned by
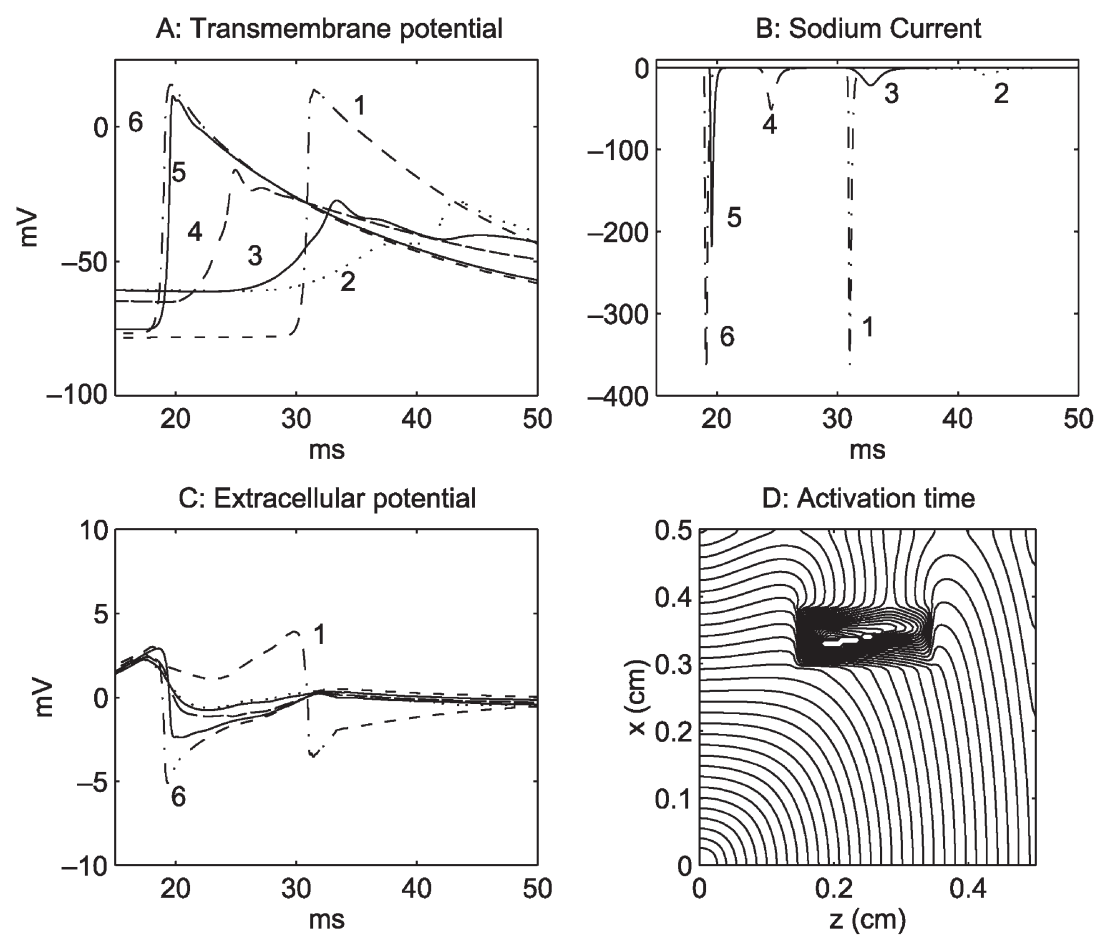

FIGURE 8 Same format as in Fig. 2. A line stimulus is applied at the origin, with a duration of 0.5 ms to initiate propagation. Discretization steps: $\Delta x=\Delta z=0.005 \mathrm{~cm}$ and $\Delta t=0.02 \mathrm{~ms}$. 
changes in $\Delta x, \Delta z$, especially when slow conduction is present. Even if no stability constraints between space and time steps exist, nevertheless, an "accuracy constraint" holds for the space steps, independent of $\Delta t$. Therefore, they have to be chosen appropriately in order to avoid numerical artifacts, especially during slow conduction. In fact, slow propagation is characterized by higher values of the space derivatives of $v$ and this explains the need for smaller space steps in order to describe accurately the rapid spatial changes of transmembrane potential.

Convergence of Galerkin semidiscretization of continuous bidomain models was studied in Ref. [31] and [32]. The several error estimates established in [31] and [32] show that the error constants depend on parameters which characterize the cardiac tissue and affect the propagation velocity. Such error estimates can be quite poor if the diffusive term of problem (1) is small compared to the reactive term, such as during slow propagation. In this situation, the error constants may be quite large and the performance of the pure Galerkin method can be poor if the space steps are not sufficiently small.

Joyner [15] described the constraint on the space step for a monodimensional propagation in terms of the space constant, defined as the number $\lambda$ such that the profile of the deviation from equilibrium of the potential, resulting from a constant subthreshold stimulus applied at a single point, is an exponential proportional to $e^{-x / \lambda}$ (see also [18] for a definition of $\lambda$ ); he found that the effects of discretization become evident when approximatively the condition

$$
\Delta x / \lambda \leq K
$$

is violated, where the constant $K$ depends on the membrane model and on the intracellular conductivities. Shaw et al. [34] observed that the constraint (8) should be respected in order for each patch of discretization to be equipotential and for the solutions to numerically converge. In other words, $\Delta x$ must be small enough so that variations in $v$ across the patch can be neglected. As the conductivities decrease, $\lambda$ decreases because $v$ varies faster with distance, i.e. $\partial v / \partial x$ is greater, and $\Delta x$ must be made smaller to preserve the equipotential condition.

It is also possible to provide a physiological explanation of this phenomenon, if we look at the waveform variations obtained using large space steps relative to propagation speed:

- reduction of propagation velocity;

- reduction of the sodium current peak;

- higher values of $\dot{v}_{\max }$;

- changes in the time profiles of the electric potentials (extracellular, transmembrane);

- reduction of extracellular potential amplitude.

These changes are very similar to the ones which would be obtained in simulations of slowed conduction with sufficiently fine space grids [33,36]. Indeed, for large $\Delta x, \Delta z$ more resistance is incorporated in a single element of decomposition, and the discretized system presents slower conduction than one should expect from the continuous model; the early part of the action potential is prolonged due to the reduced current from cell-to-cell, whereas the rate of rise of the action potential is increased because, once excitation is established, there is less current flow on to the next region to be excited. Using coarse space meshes makes the fully discrete system (4) differ from the continuous model. Thus, the discrete system can be seen as a discrete cellular model taking into account the inhomogeneous and anisotropic distribution of cell-to-cell connections.

This aspect was analyzed by Keener [18] in the case of monodimensional propagation. Shortly, we can say that the semidiscrete equations (3), deriving from the application of the finite element method associated to suitable numerical quadrature formulas as from the finite difference method, may be interpreted as a discrete cellular model where the cardiac cells are viewed as isopotential, excitable, discrete objects rather than arranged in a continuum. These discrete cells exchange a current flux and the effective total intracellular conductivity tensor among the cells is given by $\mathbf{P}^{-1} \mathbf{A}_{i}$, according to equation (3); therefore, as the space steps (appearing on the diagonal of $\mathbf{P}$ ) grow, the effective intracellular resistance increases, until a critical value is reached where conduction block occurs: the electrotonic current from a cell to the neighbors is then insufficient to raise potential above threshold and, consequently, propagation breaks off.

There are significant differences between the behavior of propagating action potential for the continuous model (1) and its semidiscrete approximation (3). Indeed, while in the continuous bidomain model increasing intracellular resistance slows propagation, but can never cause it to fail, a traveling solution of Eq. (3) may not exist for fixed $\Delta x$, $\Delta z$ and increasing intracellular resistance. Analogously, if we fix the resistance and let $\Delta x, \Delta z$ vary, we find that propagation is impossible for $\Delta x, \Delta z$ larger than a certain critical value, which is a monotonic increasing function of membrane excitability since a more excitable cell requires less stimulating current to raise it to threshold. Simulations will produce results that are correct for the continuous model only when the continuous and the semidiscrete problems have the same behavior. Any new behavior that results from a semidiscretization of the model (1) can only be due to numerical artifact. Nevertheless, the simulation will exhibit propagation failure for certain large values of $\Delta x, \Delta z$ even though this is inconsistent with the continuous model that is being approximated.

However, since conduction blocks indeed occur in real hearts, although the formation of conduction blocks in the presence of low space resolution represents a numerical artifact, it may be interesting if it is referred to the analog discrete model which takes into account the discontinuous cellular nature of the cardiac tissue. 
Further investigation should be devoted to the analysis of the role of calcium current in preventing numerical blocks in 2D simulations, using more complex membrane models. One dimensional studies [34] show that in some cases the calcium current can support slow propagation and delay the onset of block by increasing the electrotonic source current. In [33] preliminary numerical simulations of a BREJ two dimensional slab show that, although the secondary calcium current sustains depolarization, for certain choices of the discretization steps it may not be sufficient to avoid conduction blocks.

\section{CONCLUSIONS}

In this work, we analyze the complex phenomenon of electrogram fractionation, due to discontinuities and alterations in cellular coupling. Our simulations show that fractionation is independent of $I_{\mathrm{Na}}$ alterations and that it can be described by the bidomain model of cardiac tissue. Some deflections in fractionated electrograms may give nonlocal informations about the shape of damaged areas with respect to the wavefront position. When an action potential propagates at a constant velocity, a biphasic electrogram is recorded by a unipolar electrode near the surface of the tissue; on the contrary, when the action potential passes through regions with discontinuous membrane properties and heterogeneous distribution of cell-to-cell coupling resistance the corresponding electrograms deviate from normal and may present several deflections, occurring at the times when the activation wavefront crosses the discontinuity barriers. Moreover, inhomogeneities of the medium may affect also the waveforms at points located in normal regions, revealing the presence of pathological conditions at a distance.

Another issue considered in this work is the generation of conduction blocks. Our simulations show that the generation of conduction blocks depends on:

1. the employed membrane current model;

2. the intracellular conductivity properties of the tissue;

3. the resting potential value.

Moreover, propagation and block formation are also conditioned by loading effects of the underlying tissue, by the dimension of the damaged region, by fibers rotation and by the position of the injured regions with respect to the direction of the propagating wavefront.

However, in a numerical approximation of the bidomain model of cardiac tissue, conduction blocks may derive from low space resolution and represent numerical artifacts. The simulation of pathological processes and of slow conduction requires the use of spatial refinement techniques to avoid artifacts, which may occur in the numerical simulations of action potential propagation. Indeed, the use of low spatial resolution in the simulation of slow propagation in pathological tissue can induce a reduction of conduction velocity, blocks and other numerical artifacts, which are not consistent with the continuous bidomain model theory. This is due to the fact that the discrete system, obtained at the end of any approximation process, may be interpreted as a discrete model of the cardiac tissue made up of isopotential cells. In this model the effective intracellular conductivity tensor depends on the space discretization steps and the increase of these steps results in an increase of the effective intracellular resistance and can induce conduction blocks if a certain critical value is exceeded. In other words, in a discretization of continuous macroscopic cardiac tissue models, propagation failure may represent a numerical artifact.

Nevertheless, since conduction blocks indeed occur in real hearts, the formation of conduction blocks in the presence of low space resolution is interesting if it is referred to the analog discrete cellular model which, in some sense, takes into account the discontinuous nature of the cardiac tissue.

\section{Acknowledgements}

The author gratefully thanks Prof. P. Colli Franzone, Prof. E. Macchi and the late Prof. G. Di Cola for their helpful suggestions and discussions, and the anonymous referees for their precious contribution to the final version of this paper.

\section{References}

[1] Beeler, G.W. and Reuter, H. (1977) "Reconstruction of the action potential of ventricular myocardial fibres", J. Physiol. 268, $177-210$.

[2] Berbari, E.J., Lander, P., Scherlag, B.J., Lazzara, R. and Geselowitz, D.B. (1992) "Ambiguities of epicardial mapping", J. Electrocardiol. 24(suppl.), 16-20.

[3] Colli Franzone, P. and Guerri, L. (1992) "Models of the spreading of excitation in myocardial tissue", High Performance Computing in Biomedical Research.

[4] Colli Franzone, P., Guerri, L. and Rovida, S. (1990) "Wavefronts propagation in an activation model of the anisotropic cardiac tissue: asymptotic analysis and numerical simulations", J. Math. Biol. 28, $121-176$.

[5] Colli Franzone, P., Guerri, L. and Tentoni, S. (1990) "Mathematical modeling of the excitation process in myocardial tissue: influence of fiber rotation on wavefront propagation and potential field", Math. Biosci. 101, 155-235.

[6] Cooley, J.V. and Dodge, Jr, F.A. (1966) "Digital computer solutions for excitation and propagation of the nerve impulse", Biophys. J. 6, $583-599$.

[7] Di Francesco, D. and Noble, D. (1985) "A model of cardiac electrical activity incorporating ionic pumps and concentration changes", Philos. Trans. R. Soc. Lond. Ser. B 307, 353-398.

[8] Drouhard, J.P. and Roberge, F.A. (1987) "Revised formulation of the Hodgkin-Huxley representation of the sodium current in cardiac cells", Comp. Biomed. Res. 20, 333-350.

[9] Ebihara, L. and Johnson, E. (1980) "Fast sodium current in cardiac muscle. A quantitative description", Biophys. J. 32, 779-790.

[10] Ellis, W.S., Auslander, D.M. and Lesh, M.D. (1995) "Fractionated electrograms from a computer model of heterogeneously uncoupled anisotropic ventricular myocardium", Circulation 92, $1619-1626$.

[11] Gardner, P.I., Ursell, P.C., Fenoglio, J.J. and Wit, A.L. (1985) "Electrophysiologic and anatomic basis for fractionated electrograms recorded from healed myocardial infarcts", Circulation 72, 596. 
[12] Henriquez, C.S. and Plonsey, R. (1987) "Effects of resistive discontinuities on waveshape and velocity in a single cardiac fibre", Med. Biol. Eng. Comput. 25, 428-438.

[13] Hodgkin, A.L. and Huxley, A.F. (1952) "A quantitative description of membrane current and its application to conduction and excitation in nerve", J. Physiol. (Lond.) 117, 500-544.

[14] Janse, M.J. and Kléber, A.G. (1992) "Propagation of electrical activity in ischemic and infarcted myocardium as the basis of ventricular arrhythmias", J. Cardiovasc. Electrophysiol. 3(N. 1), $77-87$.

[15] Joyner, R.W. (1982) "Effects of the discrete pattern of electrical coupling on propagation through an electrical syncytium", Circ. Res. 50, 192-200.

[16] Joyner, R.W. (1981) "Mechanisms of unidirectional block in cardiac tissue", Biophys. J. 35, 113-125.

[17] Keener, J.P. (1991) "An eikonal-curvature equation for potential propagation in myocardium", J. Math. Biol. 29, 629-651.

[18] Keener, J.P. (1991) "The effects of discrete gap junction coupling on propagation in myocardium", J. Theor. Biol. 148, 49-82.

[19] Keener, J.P. (1988) "On the formation of circulating patterns of excitation in anisotropic excitable media", Math. Biosci. 26, $41-56$.

[20] Luo, C. and Rudy, Y. (1991) "A model of the ventricular cardiac action potential: depolarization, repolarization and their interaction", Circ. Res. 68, 1501-1526.

[21] Luo, C. and Rudy, Y. (1994) "A dynamic model of the cardiac ventricular action potential. I. Simulation of ionic currents and concentration changes", Circ. Res. 74, 1071-1096.

[22] Lesh, M.D., Pring, M. and Spear, J.F. (1989) "Cellular uncoupling can unmask dispersion of action potential duration in ventricular myocardium", Circ. Res. 65, 1426-1440.

[23] Lesh, M.D., Spear, J.F. and Simson, M.B. (1988) "A computer model of the electrogram: what causes fractionation?", J. Electrocardiol. 21(Suppl.), S69-S73.

[24] Marchuk, G.I. (1975) "Methods of numerical mathematics", Applications of Mathematics 2 (Springer, New York).

[25] McAllister, R.E., Noble, D. and Tsien, R.W. (1975) "Reconstruction of the electrical activity of cardiac Purkinje fibers", J. Physiol. 251, $1-59$.

[26] Morena, H., Janse, M.J., Fiolet, J.W.T., et al. (1980) “Comparison of effects of regional ischemia, hypoxia, hyperkalemia and acidosis on intracellular and extracellular potentials and metabolism in the isolated porcine heart", Circ. Res. 46, 634-646.

[27] Muller-Borer, B.J., Johnson, T.A., Gettes, L.S. and Cascio, W.E. (1995) "Failure of impulse propagation in a mathematically simulated ischemic border zone: influence of direction of propagation and cell-to-cell electrical coupling", J. Cardiovasc. Electrophysiol. 6, 1101-1112.
[28] Quarteroni, A. and Valli, A. (1994) Numerical Approximation of Partial Differential Equations (Springer, Berlin).

[29] Rudy, Y. and Quan, W.L. (1988) "The role of cellular discontinuities in reentry of cardiac excitation", Proceedings of the 10th Annual Conference of the IEEE Engineering in Medicine and Biology Society, pp 946-947.

[30] Rudy, Y. and Quan, W.L. (1987) "A model study of the effects of the discrete cellular structure on electrical propagation in cardiac tissue", Circ. Res. 61, 815-823.

[31] Sanfelici, S. (2000) "On the Galerkin Method for Semilinear Parabolic-Ordinary Systems", in Partial Differential Equations: Theory and Numerical Solution (CHAPMAN HALL/CRC Res. Notes Math. Ser., CRC Press LLC, Boca Raton, Florida), pp. 298-308.

[32] Sanfelici, S. (1998) "Convergence of the Galerkin Approximation of a Degenerate Evolution Problem in Electrocardiology", Numer. Methods Partial Differential Equations 18(2), 218-240.

[33] Sanfelici, S. (1998) "Modelli matematici del comportamento elettrico del tessuto cardiaco: metodi numerici ed applicazioni", $\mathrm{PhD}$ Thesis.

[34] Shaw, R.M. and Rudy, Y. (1997) "Electrophysiologic effects of acute myocardial ischemia. A mechanistic investigation of action potential conduction and conduction failure", Circ. Res. 80, $124-138$.

[35] El-Sherif, N., Sherlag, B.J., Lazzara, R. and Hope, R.R. (1977) "Reentrant ventricular arrhythmias in the late myocardial infarction period. I. Conduction characteristics in the infarction zone", Circulation 55, 686

[36] Spach, M.S. (1983) "The discontinuous nature of electrical propagation in cardiac muscle", Ann. Biomed. Eng. 11, 209-261, The 1982 Alza distinguished lecture.

[37] Spach, M.S., Miller, III, W.T., Geselowitz, D.B., Barr, R.C., Kootsey, J.M. and Johnson, J.A. (1981) "The discontinuous nature of propagation in normal canine cardiac muscle. Evidence for recurrent discontinuities of intracellular resistance that affect the membrane currents", Circ. Res. 48, 39-54.

[38] Spach, M.S. and Kootsey, J.M. (1985) "Relating the sodium current and conductance to the shape of transmembrane and extracellular potentials by simulation: effects of propagation boundaries", IEEE Trans. Biomed. Eng. BME-32, 743-755.

[39] Wang, Y. and Rudy, Y. (2000) "Action potential propagation in inhomogeneous cardiac tissue: safety factor considerations and ionic mechanism", Am. J. Physiol. Heart Circ. Physiol. 278, H1019-H1029.

[40] Witkowski, X., Plonsey, R., Penkoske, P.A. and Kavanagh, K.M. (1994) "Significance of inwardly directed transmembrane current in determination of local myocardial electrical activation during ventricular fibrillation", Circ. Res. 74, 507-524. 


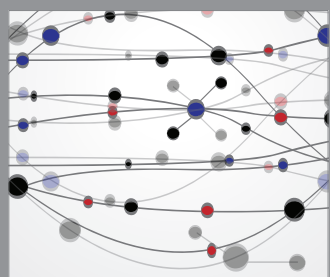

The Scientific World Journal
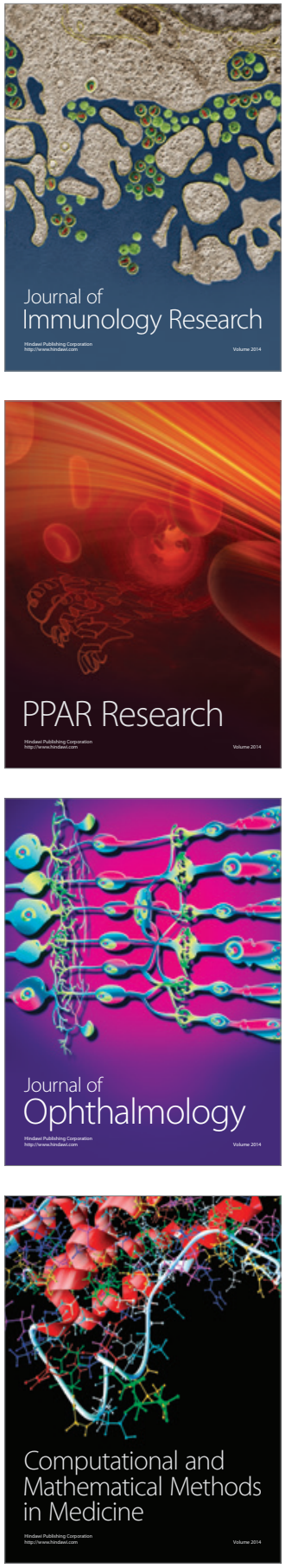

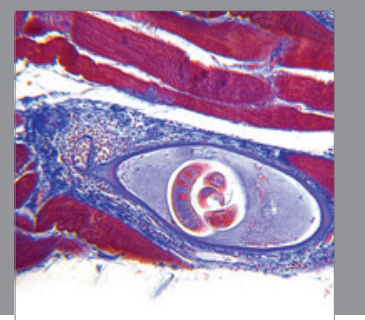

Gastroenterology

Research and Practice
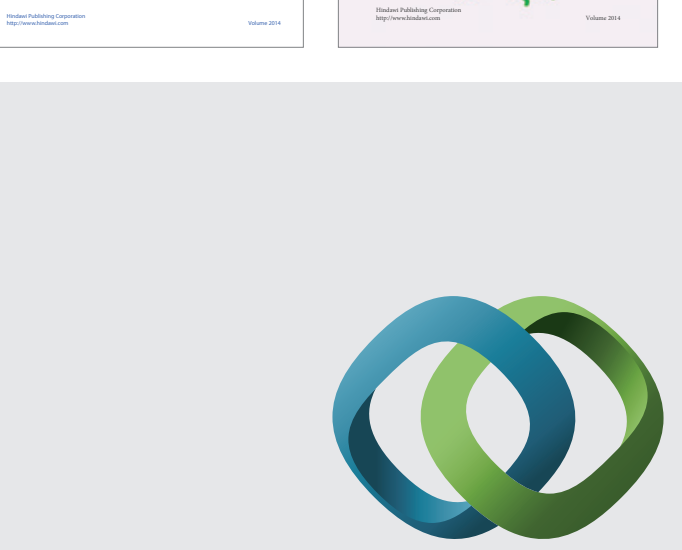

\section{Hindawi}

Submit your manuscripts at

http://www.hindawi.com
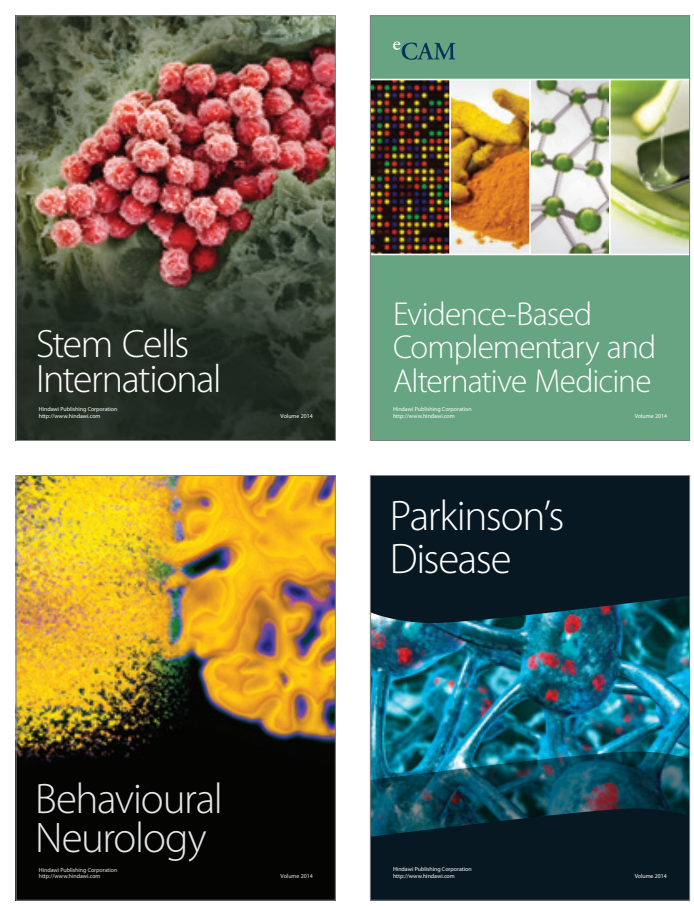

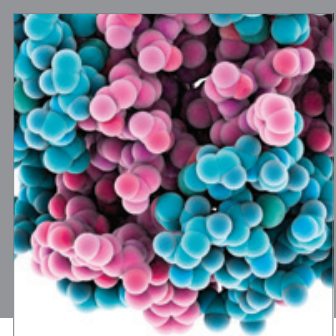

Journal of
Diabetes Research

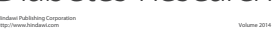

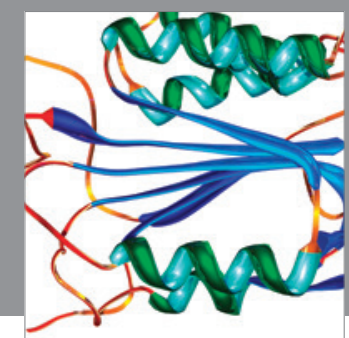

Disease Markers
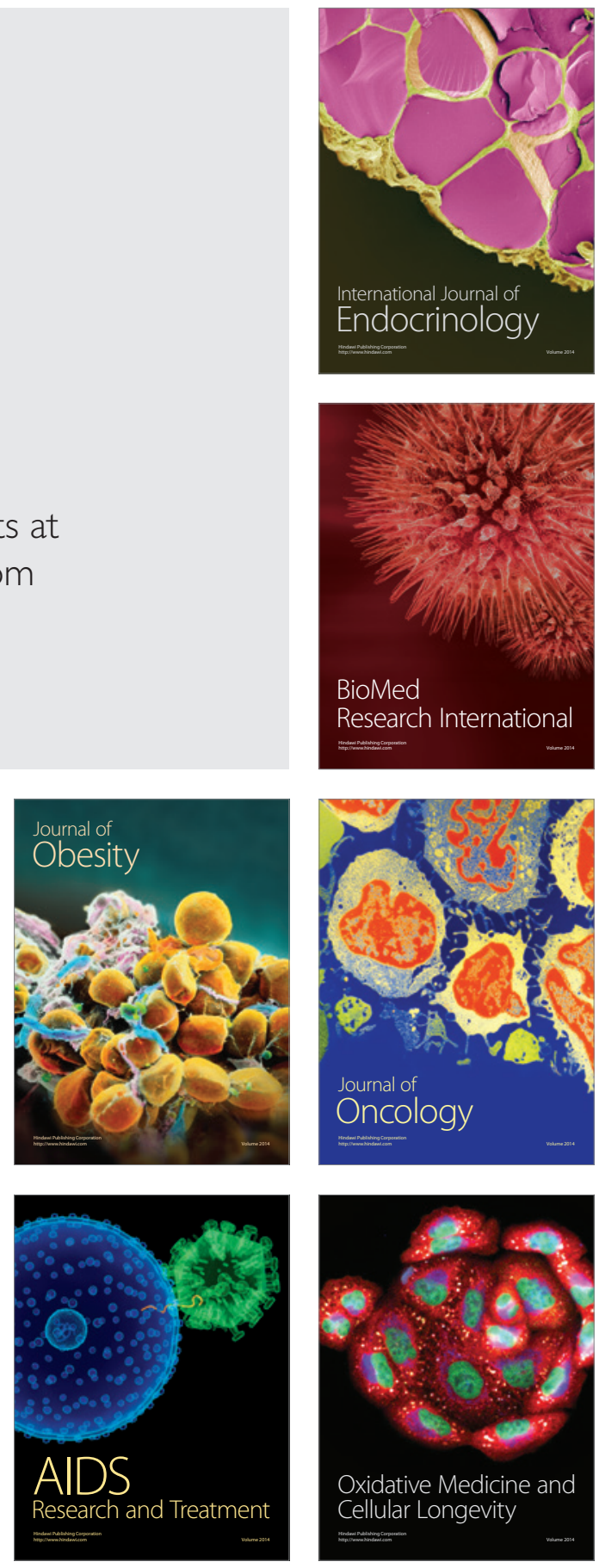